\title{
Matrices with Banded Inverses: Inversion Algorithms and Factorization of Gauss-Markov Processes
}

\author{
Aleksandar Kavčić, Member, IEEE, and José M. F. Moura, Fellow, IEEE
}

\begin{abstract}
The paper considers the inversion of full matrices whose inverses are $L$-banded. We derive a nested inversion algorithm for such matrices. Applied to a tridiagonal matrix, the algorithm provides its explicit inverse as an element-wise product (Hadamard product) of three matrices. When related to Gauss-Markov random processes (GMrp), this result provides a closed-form factored expression for the covariance matrix of a first-order GMrp. This factored form leads to the interpretation of a first-order GMrp as the product of three independent processes: a forward independent-increments process, a backward independent-increments process, and a variance-stationary process. We explore the nonuniqueness of the factorization and design it so that the forward and backward factor processes have minimum energy.

We then consider the issue of approximating general nonstationary Gaussian processes by Gauss-Markov processes under two optimality criteria: the Kullback-Leibler distance and maximum entropy. The problem reduces to approximating general covariances by covariance matrices whose inverses are banded. Our inversion result is an efficient algorithmic solution to this problem. We evaluate the information loss between the original process and its Gauss-Markov approximation.
\end{abstract}

Index Terms-Banded matrix, Cholesky decomposition, GaussMarkov processes, inhomogeneous autoregressive processes, Kullback-Leibler distance, $L$-band complement, maximum-entropy method, potential matrix, tridiagonal matrix.

\section{INTRODUCTION}

B ANDED matrices arise in numerous applications ranging from the discretization of partial differential operators to linear autoregressive models for images. When these matrices are symmetric and positive-definite, their inverses are covariance matrices of causal or noncausal Gauss-Markov random processes (GMrp). ${ }^{1}$ In this sense, they are parsimonious descriptions for GMrp's, easier to parameterize than the covariance of the process itself.

In this paper, we present an intriguing nested algorithm to invert matrices whose inverses are known to be $L$-banded. The al-

Manuscript received March 26, 1998; revised December 6, 1999. This work was supported in part by the National Science Foundation under Grants ECD8907068 and CCR-9904458. The work of J. M. F. Moura while at MIT was supported by the Army Research Office under Grant 8905-48164.

A. Kavčić is with the Division of Engineering and Applied Sciences, Harvard University, Cambridge, MA 02138 USA.

J. M. F. Moura is a visitor at LIDS, Department of Electrical Engineering and Computer Science, Massachusetts Institute of Technology, Cambridge, MA 02139 USA, on leave from Carnegie-Mellon University, Pittsburgh, PA 15213 USA.

Communicated by P. Moulin, Associate Editor for Nonparametric Estimation, Classification, and Neural Networks.

Publisher Item Identifier S 0018-9448(00)04653-8.

${ }^{1}$ These processes are also commonly referred to in the literature as Gauss-Markov random fields. gorithm applies to general matrices, not necessarily symmetric or positive-definite. The nested algorithm uses only the entries in the $L$-band of the full matrix. In other words, the entries of the matrix outside its $L$-band are not used in finding its inverse, when the inverse is $L$-banded. Again, invoking the GMrp analogy, the nested algorithm inverts the covariance matrix of a Gauss process when the process is known to be Markov.

With the insight provided by the nested algorithm, we obtain three major results: i) we derive explicit inverses for general tridiagonal matrices; ii) we present an original factorization of GMrp's as the product of three independent-increment processes; and, finally, iii) we study the approximation of Gauss processes by Gauss-Markov processes.

Explicit Inverses of Tridiagonal Matrices: Application of the nested algorithm leads to explicit expressions for the inverses of general tridiagonal matrices, i.e., $L$-banded matrices where $L=$ 1. In particular, this enables us to write, we believe for the first time, closed-form expressions for covariance matrices which are diagonalized by trigonometric transforms like the discrete cosine transform (DCT) and the discrete sine transform (DST), i.e., for which these transforms are their Karhunen-Loève transforms [1]. The explicit inverses of tridiagonal matrices are given as Hadamard (i.e., element-wise) products of three matrices.

Factorization of GMrp's: When we apply this factored decomposition to covariances of GMrp's, we obtain an original factorization of GMrp's as the product of three independent random components: a forward independent-increments process, a backward independent-increments process, and a variance-stationary random process. This factored representation is striking because it helps separate the impact of boundary conditions (initial and terminal) from the long-term behavior of the noncausal process. The forward component starts from the initial condition of the process, the backward component is a backward process started from the terminal condition, and the variance-stationary component is free at both ends. We exploit the nonuniqueness of the Hadamard factored representation to solve for the optimal factorization of the GMrp in the sense that the sum of the energies of the forward and backward components are minimal.

Approximation of Gauss Processes by Gauss-Markov Processes: We explore the role of banded matrices and the application of the nested algorithm along a third direction, namely, that of finding Gauss-Markov process approximations to general nonstationary Gauss processes. A problem related to this is that of covariance matrix extension, where it is desired to extend the covariance of a random sequence beyond a given lag. The optimal solution to either problem in the sense of the Kullback-Leibler distance or the maximum-entropy criterion corre- 
sponds to approximating the original covariance matrix by a covariance matrix whose inverse is banded. Our nested algorithm is a readily available efficient solution to this problem.

Summary of the Paper: In Section II we present the concept of the $L$-band extension of a general matrix and then our major results on the inverses of matrices with $L$-banded inverses. Theorems 1 and 2 present the nested algorithm for inversion of such matrices. Appendix A contains proofs for two lemmas presented in this section. Section III applies the nested algorithm to derive the explicit inverse for a general tridiagonal matrix. The inverse is expressed as the Hadamard product of three matrices. In Appendix B we illustrate this inverse for matrices widely used in applications. Section IV applies the results in Section III to solving the optimization problem of factoring a Gauss-Markov random process as the product of three independent processes, where the sum of the energies of the forward component and of the backward component is minimal and the third component has a covariance with prespecified form, namely, it is variance-stationary. Section V studies the approximation of general Gauss processes by Gauss-Markov processes, relates this problem to the approximation of general covariance matrices by matrices whose inverse is $L$-banded, and uses the nested inversion algorithm to provide a prompt solution to this problem. We consider both the Kullback-Leibler distance and the maximum-entropy criterion as optimality criteria. The section also presents the information loss carried by these GMrp approximations. Section VI summarizes the paper.

\section{BANDED MATRIX AlgEBRA}

\section{A. Notation}

To avoid cumbersome block-matrix expressions throughout the paper, we introduce the following notation. We denote a column vector $\underline{z}$ of $N$ elements $z_{1}, z_{2}, \cdots, z_{N}$, by

$$
\underline{z}=z_{N}^{1}=\left[\begin{array}{llll}
z_{1} & z_{2} & \cdots & z_{N}
\end{array}\right]^{T}
$$

We specify a subvector of $\underline{z}=z_{N}^{1}$ involving elements $z_{i}$ through $z_{j}$, where $1 \leq i \leq j \leq N$, by

$$
z_{j}^{i}=\left[\begin{array}{lll}
z_{i} z_{i+1} & \cdots & z_{j}
\end{array}\right]^{T} \text {. }
$$

We denote an $N \times N$ matrix $\mathbf{A}$ by $\mathbf{A}_{N}^{1}$. We will often use $\mathrm{MatLab}^{2}$ type notation. By $\mathbf{A}(i: j, k)$ we represent the column vector of the entries of the matrix $\mathbf{A}(i, k)$ through $(j, k)$,

\footnotetext{
${ }^{2}$ MatLab is a registered trademark of MathWorks
}

and likewise for the row vector $\mathbf{A}(k, i: j)$. A principal submatrix of $\mathbf{A}=\mathbf{A}_{N}^{1}$ spanning columns (and rows) $i$ through $j$ $(1 \leq i \leq j \leq N)$ is given by

$$
\mathbf{A}_{j}^{i}=\mathbf{A}(i: j, i: j)=\mathbf{A}_{N}^{1}(i: j, i: j) .
$$

We refer to a matrix as an $L$-banded matrix $(L \geq 0)$ if the elements outside the band defined by the $L$ th lower diagonal and the $L$ th upper diagonal are zero. A diagonal matrix is thus a 0-banded matrix. A tridiagonal matrix is a 1-banded matrix. Similarly, we refer to the band between (and including) the $L$ th lower and the $L$ th upper diagonal of a matrix $\mathbf{A}$ as the $L$-band of $\mathbf{A}$. The trace of matrix $\mathbf{A}$ is denoted by $\operatorname{tr} \mathbf{A}$, while its determinant is denoted by $\operatorname{det} \mathbf{A}$.

Throughout the paper, we add block-partitioned matrices in an overlapping fashion. For this purpose, we develop the following nested notation shown in (4) at the bottom of this page. The resulting matrix $\mathbf{R}$ in (4) is an $L$-banded matrix. The following $3 \times 3$ example of a 2 -banded matrix illustrates the notation in (4) (on the right-hand side we omit the zero elements)

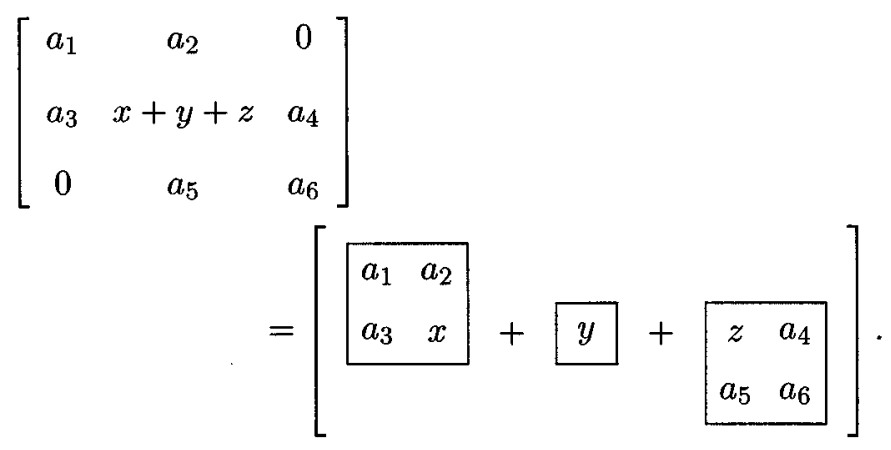

\section{B. Theorems}

The proofs of the next two lemmas are in Appendix A.

Lemma 1: Let $\mathbf{R}$ be an $N \times N$ matrix whose inverse $\mathbf{R}^{-1}$ is $L$-banded. Then

$$
\begin{aligned}
\mathbf{R}^{-1}-\left[\begin{array}{cc}
{\left[\mathbf{R}_{N-1}^{1}\right]^{-1}} & \underline{0} \\
\underline{0}^{T} & 0
\end{array}\right]= & {\left[\begin{array}{ll}
\mathbf{0} & \mathbf{0} \\
\mathbf{0} & {\left[\mathbf{R}_{N}^{N-L}\right]^{-1}}
\end{array}\right] } \\
& -\left[\begin{array}{cc}
\mathbf{0} & \mathbf{0} \\
\mathbf{0} & {\left[\begin{array}{cc}
{\left[\mathbf{R}_{N-1}^{N-L}\right]^{-1}} & \underline{0} \\
\underline{0}^{T} & 0
\end{array}\right]}
\end{array}\right.
\end{aligned}
$$

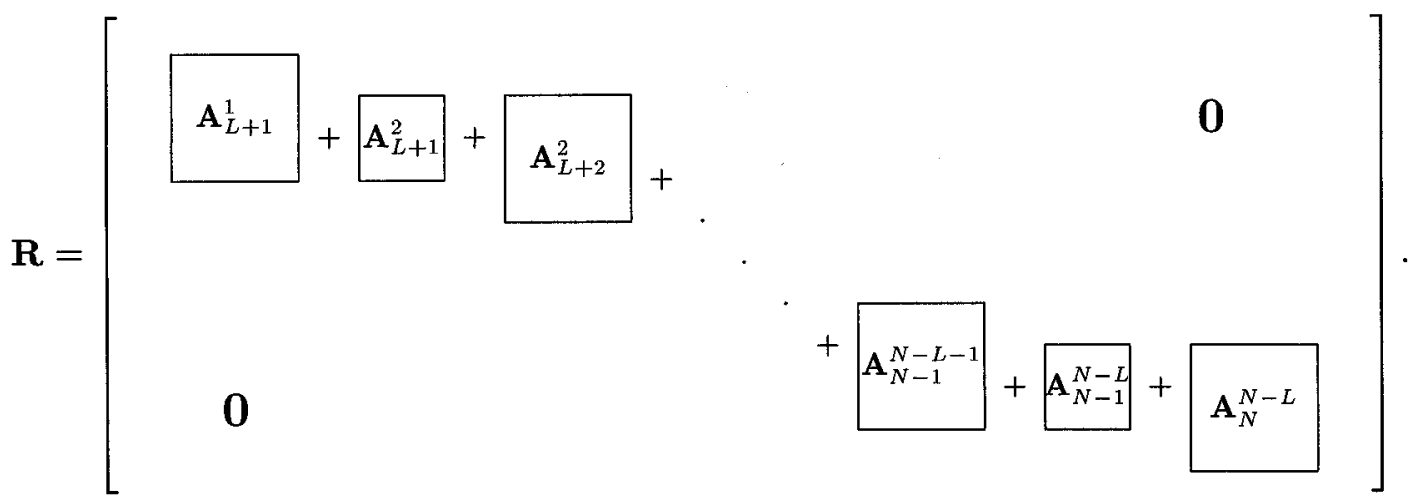


Lemma 2: If $\mathbf{R}$ has an $L$-banded inverse $\mathbf{R}^{-1}$, then the inverse $\left[\mathbf{R}_{j}^{i}\right]^{-1}$ of any principal submatrix $\mathbf{R}_{j}^{i}$ of $\mathbf{R}$ is also $L$-banded, provided that $j-i \geq L$.

Definition (L-Band Extension): The matrix $\mathbf{R}$ is called the $L$-band extension of an $N \times N$ matrix $\mathbf{C}$, if its inverse $\mathbf{R}^{-1}$ is related to $\mathbf{C}$, as shown in (7) at the bottom of this page.

Theorem 1 (Banded Matrix Inverse Theorem): $\mathbf{R}^{-1}$ is $L$ banded if and only if $\mathbf{R}$ equals its own $L$-band extension.

Proof: The if is trivially satisfied by the definition of the $L$-band extension. To prove the only if direction, we invoke the following inductive argument. Lemma 1 gives

$$
\mathbf{R}^{-1}=\left[\mathbf{R}_{N-1}^{1}-\mathbf{R}_{N-1}^{N-1}+\mathbf{R}_{N}^{N-L}\right]
$$

From Lemma 2 we have that $\left[\mathbf{R}_{N-1}^{1}\right]^{-1}$ in (8) is $L$-banded. Since $\mathbf{R}_{N-1}^{1}$ has an $L$-banded inverse, we can further apply Lemma 1 on $\left[\mathbf{R}_{N-1}^{1}\right]^{-1}$ to express $\left[\mathbf{R}_{N-1}^{1}\right]^{-1}$ in terms of $\left[\mathbf{R}_{N-2}^{1}\right]^{-1}, \quad\left[\mathbf{R}_{N-1}^{N-1}\right]^{-1}$, and $\left[\mathbf{R}_{N-2}^{N-L-1}\right]^{-1}$. Successive alternative application of Lemma 2 and Lemma 1 on

$$
\left[\mathbf{R}_{N-2}^{1}\right]^{-1},\left[\mathbf{R}_{N-3}^{1}\right]^{-1}, \cdots,\left[\mathbf{R}_{L+2}^{1}\right]^{-1}
$$

proves the theorem.

\section{Corollary 1.1: If $\mathbf{R}^{-1}$ is $L$-banded, then}

$$
\operatorname{det} \mathbf{R}=\frac{\operatorname{det} \mathbf{R}_{L+1}^{1} \cdot \operatorname{det} \mathbf{R}_{L+2}^{2} \cdots \operatorname{det} \mathbf{R}_{N}^{N-L}}{\operatorname{det} \mathbf{R}_{L+1}^{2} \cdot \operatorname{det} \mathbf{R}_{L+2}^{3} \cdots \operatorname{det} \mathbf{R}_{N-1}^{N-L}} .
$$

Proof: To prove the Corollary, recall the following formula for the product of a block-partitioned matrix:

$$
\operatorname{det}\left[\begin{array}{cc}
\mathbf{A} & \mathbf{B} \\
\mathbf{C}^{T} & \mathbf{D}
\end{array}\right]=\operatorname{det} \mathbf{A} \operatorname{det}\left[\mathbf{D}-\mathbf{C}^{T} \mathbf{A}^{-1} \mathbf{B}\right]
$$

where we assumed that $\mathbf{A}^{-1}$ exists.
We use an inductive argument. First the following hold:

$$
\begin{aligned}
\operatorname{det} \mathbf{R}=\operatorname{det} \mathbf{R}_{N}^{1} & =\operatorname{det}\left[\begin{array}{cc}
\mathbf{R}_{N-1}^{1} & \underline{B} \\
\underline{C}^{T} & r
\end{array}\right] \\
& =\operatorname{det} \mathbf{R}_{N-1}^{1} \operatorname{det}\left[r-\underline{C}^{T} \mathbf{R}_{N-1}^{1} \underline{B}\right] \\
& =\left(\operatorname{det} \mathbf{R}_{N-1}^{1}\right) H \\
\operatorname{det} \mathbf{R}_{N}^{N-L} & =\operatorname{det}\left[\begin{array}{cc}
\mathbf{R}_{N-1}^{N-L} & \underline{b} \\
\underline{c}^{T} & r
\end{array}\right] \\
& =\operatorname{det} \mathbf{R}_{N-1}^{N-L} \operatorname{det}\left[r-\underline{c}^{T} \mathbf{R}_{N-1}^{N-L} \underline{b}\right] \\
& =\left(\operatorname{det} \mathbf{R}_{N-1}^{N-L}\right) h .
\end{aligned}
$$

As shown at the end of the proof of Lemma 1 in Appendix A, the Schur complements of the last element of $\mathbf{R}$ and of $\mathbf{R}_{N}^{N-L}$, $H$ and $h$, respectively, (see (103)), are equal. Hence, from the last equation above

$$
H=h=\frac{\operatorname{det} \mathbf{R}_{N}^{N-L}}{\operatorname{det} \mathbf{R}_{N-1}^{N-L}} .
$$

Replacing this value of $H$ in (10), we get

$$
\operatorname{det} \mathbf{R}=\operatorname{det} \mathbf{R}_{N-1}^{1} \frac{\operatorname{det} \mathbf{R}_{N}^{N-L}}{\operatorname{det} \mathbf{R}_{N-1}^{N-L}} .
$$

Due to Lemma 2, we have that $\left[\mathbf{R}_{N-1}^{1}\right]^{-1}$ is $L$-banded. Due to Lemma 1, we have again

$$
\operatorname{det} \mathbf{R}_{N-1}^{1}=\operatorname{det} \mathbf{R}_{N-2}^{1} \frac{\operatorname{det} \mathbf{R}_{N-1}^{N-L-1}}{\operatorname{det} \mathbf{R}_{N-2}^{N-L-1}} .
$$

Successive application of this procedure on

$$
\operatorname{det} \mathbf{R}_{N-2}^{1}, \operatorname{det} \mathbf{R}_{N-3}^{1}, \cdots, \operatorname{det} \mathbf{R}_{L+2}^{1}
$$

leads to (11).

Corollary 1.2: Let $\mathbf{R}$ be the $L$-band complement of an $N \times$ $N$ matrix C. Then

$$
\operatorname{tr}\left(\mathbf{R}^{-1} \mathbf{C}\right)=\operatorname{tr}\left(\mathbf{C R}^{-1}\right)=N .
$$

Proof: Multiply $\mathbf{C}$ by (7) and verify that the product has ones on the main diagonal.

$$
\mathbf{R}^{-1}=\left[\begin{array}{c}
\mathbf{C}_{L+1}^{1}-\mathbf{C}_{L+1}^{2}+\mathbf{C}_{L+2}^{2}- \\
\mathbf{0} \\
\left.\quad+\mathbf{C}_{N-1}^{N-1}-\mathbf{C}_{N-1}^{N-1}+\mathbf{C}_{N}^{N-L}\right]^{-1}
\end{array}\right]
$$




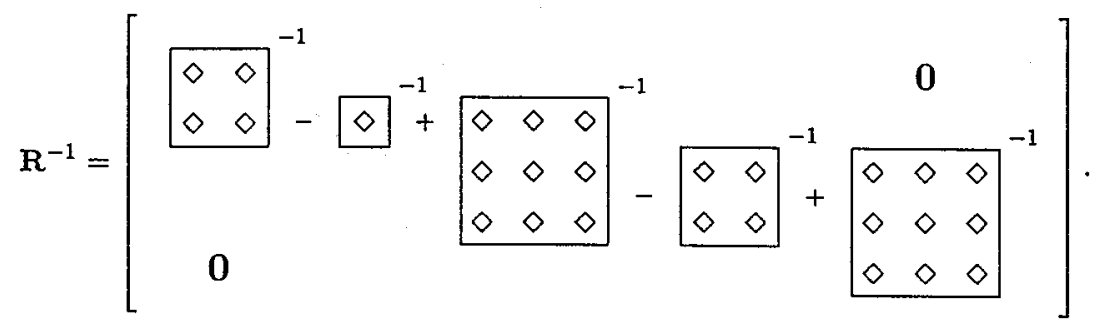

Note that Corollary 1.2 asserts only that the diagonal entries of $\left(\mathbf{R}^{-1} \mathbf{C}\right)$ are ones. It says nothing about the off-diagonal entries of $\left(\mathbf{R}^{-1} \mathbf{C}\right)$ or of $\left(\mathbf{C R}^{-1}\right)$.

The Banded Matrix Inverse Theorem (Theorem 1) can be generalized to banded matrices with nonuniform bands. We demonstrate this on an example (the proof of which is very similar to the proof of Theorem 1). Let the matrix $\mathbf{R}$ be a matrix whose inverse has the following structure:

$\mathbf{R}^{-1}=\left[\begin{array}{lllll}\diamond & \diamond & \diamond & \diamond & \diamond \\ \diamond & \diamond & \diamond & \diamond & \diamond \\ \diamond & \diamond & \diamond & \diamond & \diamond \\ \diamond & \diamond & \diamond & \diamond & \diamond \\ \diamond & \diamond & \diamond & \diamond & \diamond\end{array}\right]^{-1}=\left[\begin{array}{lllll}\square & \square & \cdot & \cdot & \cdot \\ \square & \square & \square & \square & \cdot \\ \cdot & \square & \square & \square & \square \\ \cdot & \square & 0 & \square & \square \\ \cdot & \cdot & \square & \square & 0\end{array}\right]$

where " $\diamond$ " denotes nonzero elements in the matrix $\mathbf{R}$, " $\square$ " denotes nonzero elements in the inverse matrix $\mathbf{R}^{-1}$, and "." denotes zeros. Then the inverse is given by (15) at the top of this page.

Theorem 2 (Decomposition Theorem): Let $\mathbf{C}$ be an arbitrary square matrix. There exists a unique matrix $\mathbf{R}$ whose $L$-band equals the $L$-band of $\mathbf{C}$ and whose inverse $\mathbf{R}^{-1}$ is $L$-banded. In other words, there exists a unique matrix $\mathbf{R}$ such that

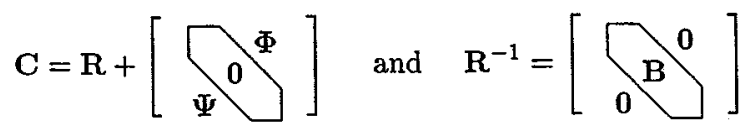

where $\mathbf{R}^{-1}$ is $L$-banded. (The matrix $\mathbf{R}$ that satisfies (16) is the $L$-band extension of $\mathbf{C}$.)

Proof: The proof follows from the uniqueness of the inverse given by the Banded Matrix Inverse Theorem (Theorem 1).

\section{UDL Decomposition}

The UDL decomposition ${ }^{3}$ of $\mathbf{R}^{-1}$ where $\mathbf{R}$ is the $L$-band extension of $\mathbf{C}$ is closely related to the nested representation of $\mathbf{R}^{-1}$ given in (7). By factoring out the right-hand side in (102)

${ }^{3}$ The UDL decomposition of a matrix $\mathbf{A}$ is the decomposition $\mathbf{A}=\mathbf{U D L}$, where $\mathbf{U}$ is upper-triangular with ones along the diagonal, $\mathbf{D}$ is diagonal, and $\mathbf{L}$ is lower-triangular with ones along the diagonal. in the proof of Lemma 1 in Appendix A, we have the following equality for a nested pair $(L+1 \leq k \leq N)$ :

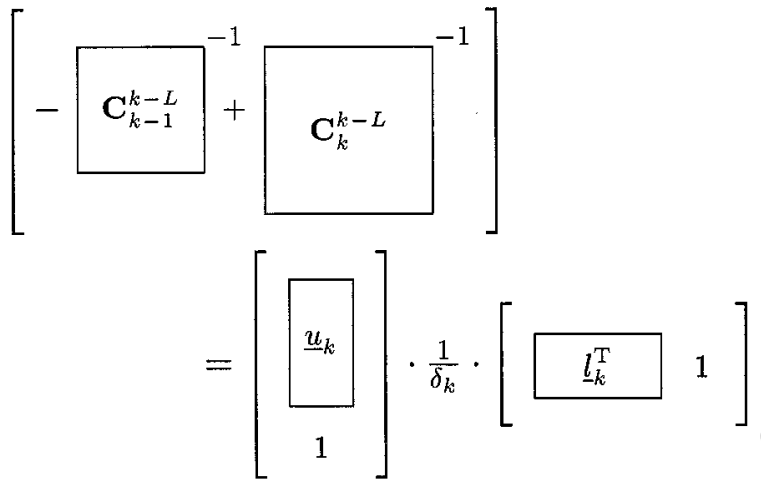

where

$$
\begin{aligned}
\underline{u}_{k} & =-\left[\mathbf{C}_{k-1}^{k-L}\right]^{-1} \cdot \mathbf{C}(k-L: k-1, k) \\
\underline{l}_{k}^{T} & =-\mathbf{C}(k, k-L: k-1) \cdot\left[\mathbf{C}_{k-1}^{k-L}\right]^{-1} \\
\delta_{k} & =\frac{\operatorname{det} \mathbf{C}_{k}^{k-L}}{\operatorname{det} \mathbf{C}_{k-1}^{k-L}} .
\end{aligned}
$$

Using (17) in (7), we find factors of the UDL decomposition $\mathbf{R}^{-1}=\mathbf{U}_{\mathbf{R}} \mathbf{D}_{\mathbf{R}} \mathbf{L}_{\mathbf{R}}$

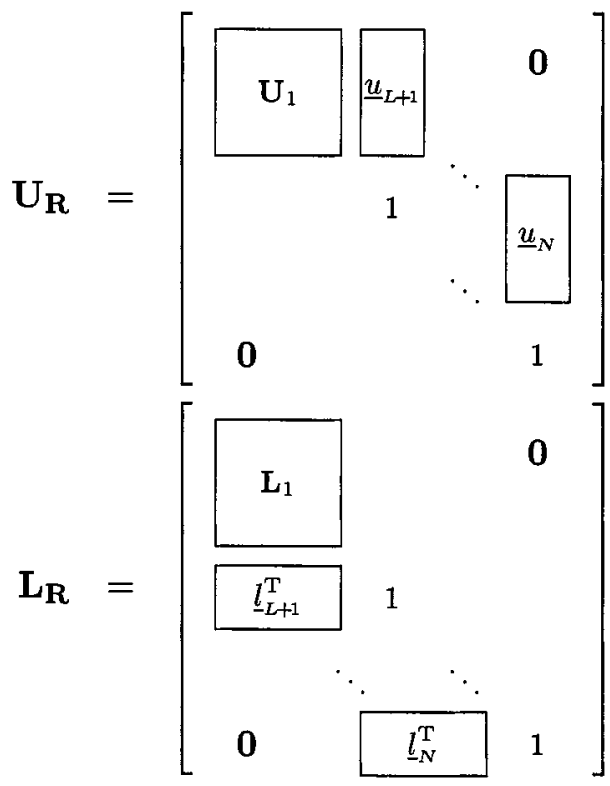


and

$$
\mathbf{D}_{\mathbf{R}}=\operatorname{diag}\left[\mathbf{D}_{1}, \frac{1}{\delta_{L+1}}, \cdots, \frac{1}{\delta_{N}}\right] .
$$

Here, $\mathbf{U}_{1}, \mathbf{D}_{1}$, and $\mathbf{L}_{1}$ are the UDL factors of $\left[\mathbf{C}_{L}^{1}\right]^{-1}=$ $\mathbf{U}_{1} \mathbf{D}_{1} \mathbf{L}_{1}$. Clearly, $\mathbf{U}_{\mathbf{R}}$ is an upper-triangular $L$-banded matrix while $\mathbf{L}_{\mathbf{R}}$ is a lower-triangular $L$-banded matrix.

\section{INVERTING TRIDIAGONAL MATRICES}

We apply the Banded Matrix Inverse Theorem, Theorem 1 in Section II, to calculate the inverse of tridiagonal banded matrices. In Section III-A, we provide a numerical procedure to find these inverses in order $\mathcal{O}\left(N^{2}\right)$. At the end of the same subsection, we discuss the generalization to $L$-banded matrices. In Section III-B, we derive closed-form expressions for the inverses of tridiagonal matrices. Examples for several frequently encountered matrices are in Appendix B.

\section{A. Algorithm for Inverting Tridiagonal Matrices}

Consider the $N \times N$ general tridiagonal matrix $\mathbf{J}^{-1}$

$$
\mathbf{J}^{-1}=\left[\begin{array}{ccccc}
q_{1} & p_{1} & & & \mathbf{0} \\
r_{1} & q_{2} & p_{2} & & \\
& r_{2} & \ddots & \ddots & \\
& & \ddots & q_{N-1} & p_{N-1} \\
\mathbf{0} & & & r_{N-1} & q_{N}
\end{array}\right] .
$$

We derive the inverse of this matrix assuming it exists. From the Banded Matrix Inverse Theorem, Theorem 1, we have (24) (at the bottom of this page), where the subblocks $\mathbf{J}_{n+1}^{n}(1 \leq n \leq$ $N-1)$ and $\mathbf{J}_{n}^{n}(2 \leq n \leq N-1)$ in (24) have the form

$$
\left[\mathbf{J}_{n+1}^{n}\right]^{-1}=\left[\begin{array}{cc}
z_{n} & p_{n} \\
r_{n} & x_{n+1}
\end{array}\right] \text { and }\left[\mathbf{J}_{n}^{n}\right]^{-1}=y_{n} .
$$

In (25), the unknown variables are $x_{n}(2 \leq n \leq N), y_{n}(2 \leq$ $n \leq N-1)$, and $z_{n}(1 \leq n \leq N-1)$. By inspection of (23)-(25), we get

$$
\begin{aligned}
z_{1} & =q_{1} \\
x_{N} & =q_{N} \\
x_{n}-y_{n}+z_{n} & =q_{n} \quad \text { for } 2 \leq n \leq N-1 .
\end{aligned}
$$

The inverses of the blocks in (25) are

$$
\begin{aligned}
\mathbf{J}_{n+1}^{n} & =\left[\begin{array}{ll}
\frac{x_{n+1}}{x_{n+1} z_{n}-p_{n} r_{n}} & \frac{-p_{n}}{x_{n+1} z_{n}-p_{n} r_{n}} \\
\frac{-r_{n}}{x_{n+1} z_{n}-p_{n} r_{n}} & \frac{z_{n}}{x_{n+1} z_{n}-p_{n} r_{n}}
\end{array}\right] \\
\mathbf{J}_{n}^{n} & =\frac{1}{y_{n}} .
\end{aligned}
$$

Since both $\mathbf{J}_{n}^{n}$ and $\mathbf{J}_{n+1}^{n+1}$ must be principal subblocks of $\mathbf{J}_{n+1}^{n}$, we get from (29)

$$
\begin{array}{rlrl}
\frac{1}{y_{n}} & =\frac{x_{n+1}}{x_{n+1} z_{n}-p_{n} r_{n}}, & & \text { for } 2 \leq n \leq N-1 \\
\frac{1}{y_{n+1}}=\frac{z_{n}}{x_{n+1} z_{n}-p_{n} r_{n}}, & \text { for } 1 \leq n \leq N-2 .
\end{array}
$$

Rearranging, we obtain

\section{forward recursion:}

from (26), (28), and (31) $\Longrightarrow z_{n+1}=q_{n+1} !-\frac{p_{n} r_{n}}{z_{n}}, z_{1}=q_{1}$,

$$
\text { for } 1 \leq n \leq N-1
$$

\section{backward recursion:}

$$
\begin{aligned}
& \text { from (27), (28), and (30) } \Longrightarrow x_{n}=q_{n}-\frac{p_{n} r_{n}}{x_{n+1}}, x_{N}=q_{N}, \\
& \text { for } 2 \leq n \leq N-1 \\
& \text { from (28) } \Longrightarrow y_{n}=x_{n}+z_{n}-q_{n}, \quad \text { for } 2 \leq n \leq N-1 .
\end{aligned}
$$

Solving canonically: from the forward recursion (32) get all $z_{n}$; from the backward recursion (33) get all $x_{n}$; from (34) and all $x_{n}$ and $z_{n}$ get all $y_{n}$. Substituting all $x_{n}, y_{n}$, and $z_{n}$ into (29), obtain the tridiagonal part, i.e., the $(L=1)$-band of $\mathbf{J}$. To get the remaining elements of $\mathbf{J}$, apply the following "filtering" method. Multiply a row of $\mathbf{J}$ with a column of $\mathbf{J}^{-1}$ and use the fact that the product must be an entry of I. For example, to get the entry $\mathbf{J}(1,3)$, multiply the first row of $\mathbf{J}$ with the third column of $\mathbf{J}^{-1}$ and equate to zero. This gives an equation for the element $\mathbf{J}(1,3)$. Repeat this going outward from the ( $L=1$ )-band of $\mathbf{J}$ sequentially sweeping all the missing diagonals of $\mathbf{J}$. The operation counts for determining the $(L=$ 1)-band of $\mathbf{J}$ and then filling the remaining of $\mathbf{J}$ are $\mathcal{O}(N)$ and $\mathcal{O}\left(N^{2}\right)$, respectively, which makes the tridiagonal matrix inversion an $\mathcal{O}\left(N^{2}\right)$ operation.

1) Inverting $L$-Banded Matrices: Let $\mathbf{A}$ be an $L$-banded matrix. Decompose $\mathbf{A}$ as $\mathbf{A}=\mathbf{Q}_{1} \mathbf{T} \mathbf{Q}_{2}^{T}$, where $\mathbf{Q}_{1}$ and $\mathbf{Q}_{2}$

$$
\mathbf{J}^{-1}=\left[\begin{array}{ccc}
\left.\mathbf{J}_{2}^{1}\right]^{-1}-\mathbf{J}_{2}^{2}+\mathbf{J}_{3}^{2}-\mathbf{0}^{-1} & \mathbf{0} \\
\mathbf{0} & \left.+\mathbf{J}_{N-1}^{N-2}-\mathbf{J}_{N-1}^{N-1}+\mathbf{J}_{N}^{N-1}\right]^{-1}
\end{array}\right]
$$


are unitary and $\mathbf{T}$ is tridiagonal. The complexity of this decomposition is $\mathcal{O}\left(L^{2} N\right)$ since both $\mathbf{Q}_{1}$ and $\mathbf{Q}_{2}$ can be obtained in closed form with $N-L$ Householder reflections of size $L \times L$ [2]. The inverse is then given by $\mathbf{A}^{-1}=\mathbf{Q}_{2} \mathbf{T}^{-1} \mathbf{Q}_{1}^{T}$, where we can use the method described in Section III-A to invert a tridiagonal matrix $\mathbf{T}$. The operation counts for the factorization $\mathbf{A}=\mathbf{Q}_{1} \mathbf{T} \mathbf{Q}_{2}^{T}$, the inversion $\mathbf{T}^{-1}$, and the multiplication $\mathbf{A}^{-1}=\mathbf{Q}_{2} \mathbf{T}^{-1} \mathbf{Q}_{1}^{T}$ are $\mathcal{O}\left(L^{2} N\right), \mathcal{O}\left(N^{2}\right)$, and $\mathbf{O}\left(L N^{2}\right)$, respectively, making the $L$-banded matrix inversion an $\mathcal{O}\left(L N^{2}\right)$ complexity operation. This is useful when $L \ll N$.

\section{B. Explicit Inverses of Tridiagonal Matrices}

We now use the forward and backward recursions in (32)-(34) to derive explicit inverses for tridiagonal matrices. Define the following forward-recursing and backward-recursing determinants of $\mathbf{J}^{-1}$ in (23):

$$
\begin{aligned}
& f_{0}=1 \quad \text { and } \quad b_{0}=1 \\
& f_{1}=q_{1} \quad \text { and } \quad b_{1}=q_{N} \\
& f_{n}=\operatorname{det}\left[\mathbf{J}^{-1}\right]_{n}^{1} \quad \text { and } \quad b_{n}=\operatorname{det}\left[\mathbf{J}^{-1}\right]_{N}^{N-n+1} \text {, } \\
& \text { for } 1 \leq n \leq N \\
& f_{N}=b_{N} .
\end{aligned}
$$

Applying the Jacobi expansion to evaluate these determinants, gives rise to the following Jacobi determinant recursion formulas:

$f_{n}=q_{n} f_{n-1}-r_{n-1} p_{n-1} f_{n-2}, \quad$ for $2 \leq n \leq N$

$b_{n}=q_{N-n+1} b_{n-1}-r_{N-n+1} p_{N-n+1} b_{n-2}, \quad$ for $2 \leq n \leq N$.

Combining these with the recursions (32)-(34), we get

from (39) and (32) $\Longrightarrow$

$$
z_{n}=\frac{f_{n}}{f_{n-1}}, \quad \text { for } 1 \leq n \leq N-1
$$

from (40) and (33) $\Longrightarrow$

$$
x_{n}=\frac{b_{N-n+1}}{b_{N-n}}, \quad \text { for } 2 \leq n \leq N
$$

(32), (33), (41), and (42) $\Longrightarrow$

$$
\begin{aligned}
-q_{n} b_{N-n} f_{n-1}= & -q_{n+1} f_{n} b_{N-n-1}+f_{n+1} b_{N-n-1} \\
& -b_{N-n+1} f_{n-1}, \quad \text { for } 1 \leq n \leq N-1
\end{aligned}
$$

from (34), (41), and (42) $\Longrightarrow$

$$
\begin{array}{r}
y_{n}=\frac{b_{N-n+1} f_{n-1}+f_{n} b_{N-n}-q_{n} b_{N-n} f_{n-1}}{b_{N-n} f_{n-1}}, \\
\text { for } 2 \leq n \leq N-1 .
\end{array}
$$

Applying the recursion (43) successively to (44), we get

$$
\begin{aligned}
y_{n} & =\frac{f_{N-1} b_{1}+f_{N} b_{0}-q_{N} f_{N-1} b_{0}}{b_{N-n} f_{n-1}} \\
& =\frac{f_{N}}{b_{N-n} f_{n-1}}, \quad \text { for } 2 \leq n \leq N-1
\end{aligned}
$$

where we used $b_{1}=q_{N}$ and $b_{0}=1$. We then get

from (41), (42), (45), and (29) $\Longrightarrow$

$$
\mathbf{J}_{n+1}^{n}=\frac{1}{f_{N}}\left[\begin{array}{cc}
b_{N-n} f_{n-1} & -p_{n} b_{N-n-1} f_{n-1} \\
-r_{n} b_{N-n-1} f_{n-1} & b_{N-n-1} f_{n}
\end{array}\right],
$$

i.e., the tridiagonal ( $L=1)$-band of $\mathbf{J}$. Using the "filtering" approach for filling the rest of the matrix $\mathbf{J}$ as described in Section III-A, we get the closed-form solution for the $(i, j)$ th element of $\mathbf{J}$

$$
\mathbf{J}(i, j)= \begin{cases}\frac{1}{f_{N}} f_{i-1} b_{N-j} \prod_{l=i}^{j-1}\left(-p_{l}\right), & \text { for } j>i \\ \frac{1}{f_{N}} f_{i-1} b_{N-i}, & \text { for } i=j \\ \frac{1}{f_{N}} f_{j-1} b_{N-i} \prod_{l=j}^{i-1}\left(-r_{l}\right), & \text { for } i>j\end{cases}
$$

Collecting in matrix format, the tridiagonal matrix $\mathbf{J}^{-1}$ has the inverse

$$
\begin{array}{r}
\mathbf{J}=\frac{1}{\sqrt{f_{N}}}\left[\begin{array}{ccccc}
f_{0} & f_{0} & f_{0} & \cdots & f_{0} \\
f_{0} & f_{1} & f_{1} & \cdots & f_{1} \\
f_{0} & f_{1} & f_{2} & \cdots & f_{2} \\
\vdots & \vdots & \vdots & \ddots & \vdots \\
f_{0} & f_{1} & f_{2} & \cdots & f_{N-1}
\end{array}\right] \\
\odot \frac{1}{\sqrt{f_{N}}}\left[\begin{array}{ccccc}
b_{N-1} & \cdots & b_{2} & b_{1} & b_{0} \\
\vdots & \ddots & \vdots & \vdots & \vdots \\
b_{2} & \cdots & b_{2} & b_{1} & b_{0} \\
b_{1} & \cdots & b_{1} & b_{1} & b_{0} \\
b_{0} & \cdots & b_{0} & b_{0} & b_{0}
\end{array}\right]
\end{array}
$$

$$
\odot\left[\begin{array}{ccccc}
1 & -p_{1} & p_{1} p_{2} & \cdots & \prod_{l=1}^{N-1}\left(-p_{l}\right) \\
-r_{1} & 1 & -p_{2} & \cdots & \prod_{l=2}^{N-1}\left(-p_{l}\right) \\
r_{1} r_{2} & -r_{2} & 1 & \cdots & \prod_{l=3}^{N-1}\left(-p_{l}\right) \\
\vdots & \vdots & \vdots & \ddots & \vdots \\
\prod_{l=1}^{N-1}\left(-r_{l}\right) & \prod_{l=2}^{N-1}\left(-r_{l}\right) & \prod_{l=3}^{N-1}\left(-r_{l}\right) & \cdots & 1
\end{array}\right]
$$

where $\odot$ stands for the Hadamard product, i.e., the element-wise matrix product. In Appendix B, a number of frequently encountered tridiagonal matrices are examined.

Nonuniqueness of the Factorization: In Section IV we explore the representation given by (48) to derive equivalent factored representations for Gauss-Markov processes. Before doing that, we show that the factored representation (48) is not unique. Because of the relation with Gauss-Markov processes that we study in Section IV, we restrict the discussion now to symmetric, positive-definite matrices J. Accordingly, we take $r_{i}=p_{i}$ in the expression of $\mathbf{J}^{-1}$ in (23). 
In fact, there are many possible equivalent representations

$$
\begin{aligned}
& \mathbf{J}=\overbrace{\left[\begin{array}{ccccc}
\sigma_{f, 0}^{2} & \sigma_{f, 0}^{2} & \sigma_{f, 0}^{2} & \cdots & \sigma_{f, 0}^{2} \\
\sigma_{f, 0}^{2} & \sigma_{f, 1}^{2} & \sigma_{f, 1}^{2} & \cdots & \sigma_{f, 1}^{2} \\
\sigma_{f, 0}^{2} & \sigma_{f, 1}^{2} & \sigma_{f, 2}^{2} & \cdots & \sigma_{f, 2}^{2} \\
\vdots & \vdots & \vdots & \ddots & \vdots \\
\sigma_{f, 0}^{2} & \sigma_{f, 1}^{2} & \sigma_{f, 2}^{2} & \cdots & \sigma_{f, N-1}^{2}
\end{array}\right]}^{\mathbf{w}_{f}} \\
& \overbrace{\left[\begin{array}{ccccc}
\sigma_{b, N-1}^{2} & \cdots & \sigma_{b, 2}^{2} & \sigma_{b, 1}^{2} & \sigma_{b, 0}^{2} \\
\vdots & \ddots & \vdots & \vdots & \vdots \\
\sigma_{b, 2}^{2} & \cdots & \sigma_{b, 2}^{2} & \sigma_{b, 1}^{2} & \sigma_{b, 0}^{2} \\
\sigma_{b, 1}^{2} & \cdots & \sigma_{b, 1}^{2} & \sigma_{b, 1}^{2} & \sigma_{b, 0}^{2} \\
\sigma_{b, 0}^{2} & \cdots & \sigma_{b, 0}^{2} & \sigma_{b, 0}^{2} & \sigma_{b, 0}^{2}
\end{array}\right]}^{\mathbf{W}_{b}}
\end{aligned}
$$

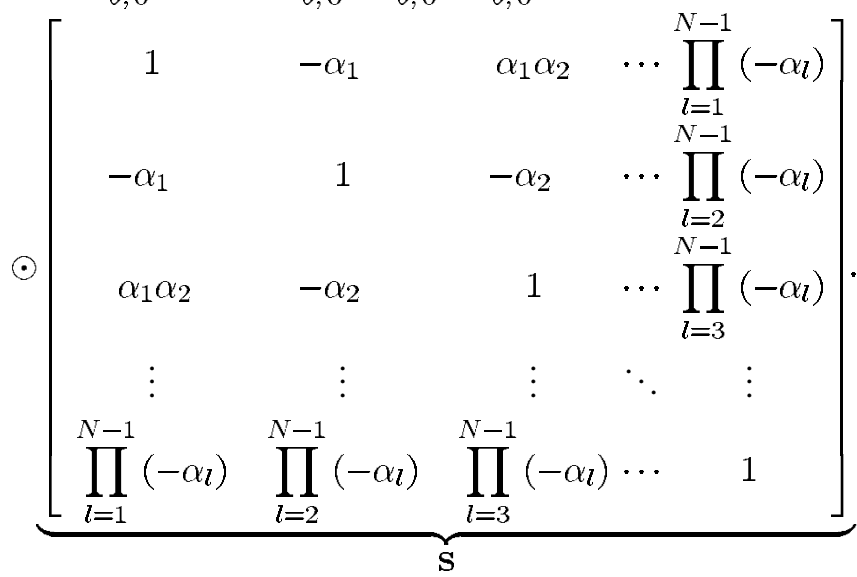

The factorization (49) is equivalent to (48) if the entries $\sigma_{f, i}^{2}$ in $\mathbf{W}_{f}, \sigma_{b, i}^{2}$ in $\mathbf{W}_{b}$, and $\alpha_{i}$ in $\mathbf{S}$ satisfy the following set of conditions:

$$
\begin{aligned}
\frac{1}{f_{N}} f_{i} b_{N-i-1} & =\sigma_{f, i}^{2} \sigma_{b, N-i-1}^{2}, & & \text { for } 0 \leq i \leq N-1 \\
\frac{1}{f_{N}} f_{i} b_{N-i-2} p_{i+1} & =\sigma_{f, i}^{2} \sigma_{b, N-i-2}^{2} \alpha_{i+1}, & & \text { for } 0 \leq i \leq N-2 .
\end{aligned}
$$

\section{FACTORED REPRESENTATIONS OF GAUSS-MARKOV PROCESSES}

We turn our attention now to symmetric positive-definite tridiagonal matrices $\mathbf{J}^{-1}$. Accordingly, $p_{n}=r_{n}$ in (23). Such $\mathbf{J}^{-1}$ are potential matrices, i.e., inverses of covariance matrices, of first order acausal nonstationary Gauss-Markov random processes (GMrp) $z_{n}[3]$ defined on a finite lattice. Conversely, every first-order acausal GMrp has a covariance matrix whose inverse is tridiagonal [3]. For simplicity, we take $z_{n}$ to be zero mean. Acausal or noncausal GMrp's can be described by difference equations. For $z_{n}$ with potential matrix $\mathbf{J}^{-1}$ given by (23), we have

$$
z_{n}+p_{n-1} z_{n-1}+p_{n+1} z_{n+1}=\epsilon_{n}, \quad 0 \leq n \leq N-1 .
$$

This difference equation is adjusted at $n=0$ and $n=N-1$ where we need to specify two boundary conditions: a left ("ini- tial") boundary condition $z_{-1}$ and a right ("terminal") boundary condition $z_{N}$. We assume that (51) is the minimum mean-square error realization of the GMrp, [4]. Stacking the processes $z_{n}$ and $\epsilon_{n}$ in $N$-dimensional vectors $\underline{z}$ and $\underline{\epsilon}$, (51) leads to the matrix equation

$$
\mathbf{J}^{-1} \underline{z}=\underline{\epsilon} .
$$

The driving noise $\epsilon_{n}$ is correlated, its covariance being

$$
\Sigma_{\epsilon}=\mathbf{J}^{-1} .
$$

As mentioned before, the covariance of $\underline{z}$ is

$$
\boldsymbol{\Sigma}_{z}=\mathbf{J} \text {. }
$$

Factored Representations: Equation (48), and more generally (49), factors the covariance matrix $\mathbf{J}$ as the Hadamard product of three matrices $\mathbf{W}_{f}, \mathbf{W}_{b}$, and $\mathbf{S}$, so that each entry of $\mathbf{J}$ is the product of three quantities. When these three matrices are positive-semidefinite, it follows that the zero-mean GMrp $z_{n}, 0 \leq n \leq N-1$, with covariance matrix $\mathbf{J}$ can be represented as a product of three uncorrelated processes

$$
z_{n}=w_{f, n} \cdot x_{n} \cdot w_{b, N-n-1}, \quad 0 \leq n \leq N-1
$$

where $\mathbf{W}_{f}$ is the covariance matrix of $w_{f, n}, 0 \leq n \leq N-1$, $\mathbf{W}_{b}$ is the covariance matrix of $w_{b, N-n-1}, 0 \leq n \leq N-1$, and $\mathbf{S}$ is the covariance matrix of $x_{n}, 0 \leq n \leq N-1$. We now explore the factorization given by (49) and what it says about the representation (53) of the GMrp.

The three factors in (49) are highly structured. The first factor $\mathbf{W}_{f}$ with its telescoping pattern has the structure of a (forward) Wiener matrix. The second factor $\mathbf{W}_{b}$ has the structure of a backward Wiener matrix. Finally, the third factor $\mathbf{S}$ has normalized diagonal entries of 1 , and generalizes the structure of a geometric matrix. The general entry $(i, j)$ of a geometric matrix is of the form $\alpha^{|i-j|}$.

Assuming that all these factors are positive-semidefinite (Section IV-A addresses this issue), we associate to each of these matrices special classes of processes. To a Wiener matrix we can associate a (zero-mean) independent-increment process whose covariance is that matrix. With $\mathbf{W}_{f}$, we associate the forward independent-increment process $w_{f, n}$ that satisfies the following description:

$$
\begin{aligned}
w_{f, n} & =w_{f, n-1}+u_{n}, \quad 0 \leq n \leq N-1 \\
\sigma_{u, n}^{2} & =E\left[u_{n}^{2}\right] \\
\sigma_{f, n}^{2} & =E\left[w_{f, n}^{2}\right]=\sigma_{f, n-1}^{2}+\sigma_{u, n}^{2} \\
\sigma_{f,-1}^{2} & =0 .
\end{aligned}
$$

The increments of $w_{f, n}$ are the driving process $u_{n}$ which is a zero mean, white process. The process $w_{f, n}$ is started from zero.

Similarly, we associate to the second factor $\mathbf{W}_{b}$ a backward independent-increment process $w_{b, N-n-1}$ described by the difference equation

$$
\begin{aligned}
w_{b, n} & =w_{b, n-1}+v_{n}, \quad 0 \leq n \leq N-1 \\
\sigma_{v, n}^{2} & =E\left[v_{n}^{2}\right] \\
\sigma_{b, n}^{2} & =E\left[w_{b, n}^{2}\right]=\sigma_{b, n-1}^{2}+\sigma_{v, n}^{2} \\
\sigma_{b,-1}^{2} & =0 .
\end{aligned}
$$


This is a backward process, started from zero initial condition. The increments of $w_{b, n}$ are $v_{n}$, a zero-mean white process.

Finally, to interpret the third factor $\mathbf{S}$, we find the UDL decomposition of $\mathbf{S}^{-1}$ as

$$
\mathbf{S}^{-1}=\mathbf{L}^{T} \mathbf{D L}
$$

where $\mathbf{L}$ is the lower-triangular matrix

$$
\mathbf{L}=\left[\begin{array}{cccccc}
1 & 0 & \cdots & \cdots & 0 & 0 \\
\alpha_{1} & 1 & \cdots & \cdots & 0 & 0 \\
0 & \alpha_{2} & \ddots & \cdots & 0 & 0 \\
\vdots & \vdots & \ddots & \ddots & \vdots & \vdots \\
0 & 0 & \cdots & \cdots & 1 & 0 \\
0 & 0 & \cdots & \cdots & \alpha_{N-1} & 1
\end{array}\right]
$$

and $\mathrm{D}$ is a diagonal matrix where

$$
\mathbf{D}^{-1}=\operatorname{diag}\left[1,1-\alpha_{1}^{2}, \cdots, 1-\alpha_{N-1}^{2}\right] .
$$

From (63) and (64), we associate with $\mathbf{S}$ the scalar, first-order, variance-stationary Markov random process

$$
x_{n}=-\alpha_{n} x_{n-1}+\eta_{n}
$$

where

$$
\begin{aligned}
\alpha_{0} & =0 \\
\sigma_{\eta, n}^{2} & =E\left[\eta_{n}^{2}\right]=1-\alpha_{n-1}^{2} \\
\sigma_{x, n}^{2} & =E\left[x_{n}^{2}\right]=1 .
\end{aligned}
$$

The above developments suggest that an acausal GMrp $z_{n}$ whose associated covariance $\mathbf{J}$ has a tridiagonal inverse can be represented by the product of three Markov processes: a forward independent-increment process $w_{f, n}$, a backward independent-increment process $w_{b}, N-n-1$, and a variance-stationary process $x_{n}$ (we address the existence of this factorization rigorously in Section IV-A). This representation is interesting because it describes the acausal process $z_{n}$ pinned by two boundary conditions, one specified at $n=-1$ and the other at $n=N$, in terms of the causal forward process which is pinned at the initial time but free at the end time, the anticausal backward process which is free at the initial time (of the original process) but pinned at the end time (of the original process), and, finally, a process that captures the long-term behavior of the original process (no pinning at either initial or terminal times). We will refer to these three processes associated with the original GMrp $z_{n}$ as the forward boundary process, the backward boundary process, and the essential component process (their respective covariance matrices are $\mathbf{W}_{f}, \mathbf{W}_{b}$, and $\mathbf{S})$. Likewise, we refer to the increment processes $u_{n}$ and $v_{n}$ as the forward and backward boundary error processes, respectively.

The factorized representation of the GMrp $z_{n}$ in (53) is not unique since the factorization of its covariance matrix in (49) is not unique either (in fact, in some cases it is not possible to find factors $\mathbf{W}_{f}, \mathbf{W}_{b}$, and $\mathbf{S}$ that are all positive-semidefinite; see Section IV-A for details). We can exploit this nonuniqueness to derive factorizations that are "most interesting" in some sense.
In particular, defining the total forward and backward boundary error energy as

$$
\sigma_{e}^{2}=\sum_{i=0}^{N-1}\left(\sigma_{u, i}^{2}+\sigma_{v, i}^{2}\right)=\sigma_{f, N-1}^{2}+\sigma_{b, N-1}^{2}
$$

we look for factorizations that minimize this total boundary error energy. We develop this concept further in the next subsection.

\section{A. Canonical Factored Representations}

We start by setting up the problem. We are given the $N \times$ $N$ symmetric positive-definite tridiagonal matrix $\mathbf{J}^{-1}$, see (23) with $r_{n}=p_{n}$. We look for the parameters

$$
\left\{\sigma_{f, n}, \sigma_{b, n}, \alpha_{n}\right\}_{0 \leq n \leq N-1}
$$

such that the representation in (49) is satisfied, and the total boundary error energy $\sigma_{e}^{2}$ is minimized. In other words, we search for a very specific factored representation of the process $z_{n}$ associated with $\mathbf{J}$, one where the sum of the forward and backward error processes energy is minimal.

This is a constrained optimization problem, since the parameters $\left\{\sigma_{f, n}^{2}, \sigma_{b, n}^{2}, \alpha_{n}\right\}_{0 \leq n \leq N-1}$ satisfy the relations in (50). Further, to satisfy the positive semidefiniteness of $\mathbf{W}_{f}$ and $\mathbf{W}_{b}$, the sequences $\left\{\sigma_{f, n}^{2}\right\}_{0 \leq n \leq N-1},\left\{\sigma_{b, n}^{2}\right\}_{0 \leq n \leq N-1}$ are monotonically nondecreasing and positive, while $\alpha_{i}, 1 \leq i \leq N-1$, are such that $\mathbf{S}^{-1}>0$ as tested, for example, by Sylvester's conditions.

We manipulate (50) to derive more explicit relations. Evaluating the top equation in (50) at $i=N-1$, and dividing the top equation by the bottom equation in (50), we get the following relations for the backward variance parameters:

$$
\begin{aligned}
\sigma_{b, 0}^{2} & =\frac{1}{f_{N}} \cdot \frac{f_{N-1} b_{0}}{\sigma_{f, N-1}^{2}} \\
\frac{\sigma_{b, N-(i+1)}^{2}}{\sigma_{b, N-(i+2)}^{2}}= & \left(\frac{\alpha_{i+1}}{p_{i+1}}\right) \frac{b_{N-(i+1)}}{b_{N-(i+2)}}, \\
& \text { for } 0 \leq i \leq N-2 .
\end{aligned}
$$

Similarly, evaluating the top equation (50) at $i=0$, and dividing the top equation by the bottom equation (rewritten at $i-1$ ) in (50), we get the relations for the forward variance parameters

$$
\begin{aligned}
\sigma_{f, 0}^{2} & =\frac{1}{f_{N}} \cdot \frac{f_{0} b_{N-1}}{\sigma_{b, N-1}^{2}} \\
\frac{\sigma_{f, i}^{2}}{\sigma_{f, i-1}^{2}} & =\left(\frac{\alpha_{i}}{p_{i}}\right) \frac{f_{i}}{f_{i-1}}, \quad \text { for } 1 \leq i \leq N-1 .
\end{aligned}
$$

Because all $\sigma_{f, n}^{2}, \sigma_{b, n}^{2}, f_{n}$, and $b_{n}$ are required to be positive, we conclude from (71) to (73) that

$$
\frac{\alpha_{i}}{p_{i}} \geq 0 \text {. }
$$

From (56) and (60), to guarantee positive semidefiniteness of $\mathbf{W}_{f}$ and $\mathbf{W}_{b}$, we must have

$$
\begin{aligned}
& \frac{\sigma_{f, i}^{2}}{\sigma_{f, i-1}^{2}} \geq 1 \\
& \frac{\sigma_{b, i}^{2}}{\sigma_{b, i-1}^{2}} \geq 1
\end{aligned}
$$


Using these equations with (71) and (73), we get

$$
\begin{array}{ll}
\frac{\alpha_{i}}{p_{i}} \geq \frac{f_{i-1}}{f_{i}}, & 1 \leq i \leq N-1 \\
\frac{\alpha_{i}}{p_{i}} \geq \frac{b_{N-(i+1)}}{b_{N-i}}, & 1 \leq i \leq N-1 .
\end{array}
$$

Solving recursively either (71) or (73), we get the constraint

$$
\sigma_{f, N-1}^{2} \sigma_{b, N-1}^{2}=\left[\prod_{i=1}^{N-1} \frac{\alpha_{i}}{p_{i}}\right] \frac{b_{N-1} f_{N-1}}{f_{N}} .
$$

Canonical Realization: Combining these relations, we restate the optimization problem as follows.

$$
\begin{gathered}
\text { minimize } \quad \sigma_{e}^{2}=\sum_{i=0}^{N-1}\left(\sigma_{u, i}^{2}+\sigma_{v, i}^{2}\right)=\sigma_{f, N-1}^{2}+\sigma_{b, N-1}^{2} \\
\text { subject to } \quad \sigma_{f, N-1}^{2} \sigma_{b, N-1}^{2}=\left[\prod_{i=1}^{N-1} \frac{\alpha_{i}}{p_{i}}\right] \frac{b_{N-1} f_{N-1}}{f_{N}} \\
\qquad \frac{\sigma_{b, N-(i+1)}^{2}}{\sigma_{b, N-(i+2)}^{2}}=\left(\frac{\alpha_{i+1}}{p_{i+1}}\right) \frac{b_{N-(i+1)}}{b_{N-(i+2)}} \geq 1 \\
\frac{\sigma_{f, i}^{2}}{\sigma_{f, i-1}^{2}}=\left(\frac{\alpha_{i}}{p_{i}}\right) \frac{f_{i}}{f_{i-1}} \geq 1 \leq N-2 \\
\alpha_{i} \text { such that } \mathbf{S}^{-1}>0, \quad 1 \leq i \leq N-1 \leq 1
\end{gathered}
$$

Solution: We solve the optimization problem (79)-(83) by first disregarding condition (83). Then we show that this solution is either the solution to the whole optimization problem including condition (83), or there exists no solution to the optimization as posed in (79)-(83). Denote the right-hand side of (80) by $\gamma$. Replacing the value of $\sigma_{b, N-1}^{2}$ from (80) into (79), we rewrite $\sigma_{e}^{2}$ as

$$
\sigma_{e}^{2}=\sigma_{f, N-1}^{2}+\frac{\gamma}{\sigma_{f, N-1}^{2}}
$$

We minimize this quantity subject to $\sigma_{f, N-1}^{2}>0$. Equating the first derivative to zero, and using the positive constraint, we get

$$
\sigma_{f, N-1}^{2}=\sqrt{\gamma}=\sqrt{\left[\prod_{i=1}^{N-1} \frac{\alpha_{i}}{p_{i}}\right] \frac{b_{N-1} f_{N-1}}{f_{N}}}
$$

By taking the second derivative, one can check that this unique extreme is, in fact, the minimum. From (80) it follows that

$$
\sigma_{b, N-1}^{2}=\sigma_{f, N-1}^{2}=\sqrt{\left[\prod_{i=1}^{N-1} \frac{\alpha_{i}}{p_{i}}\right] \frac{b_{N-1} f_{N-1}}{f_{N}}} .
$$

To minimize $\sigma_{e}^{2}=2 \sqrt{\gamma}$, we minimize $\gamma^{2}$ by minimizing

$$
\prod_{i=1}^{N-1} \frac{\alpha_{i}}{p_{i}}
$$

Since each factor in (85) satisfies also (81) and (82), the optimal $\left\{\alpha_{i}\right\}$ are

$$
\alpha_{i}=p_{i} \cdot \max \left\{\frac{f_{i-1}}{f_{i}}, \frac{b_{N-(i+1)}}{b_{N-i}}\right\}, \quad 1 \leq i \leq N-1 .
$$

Condition (83) is now satisfied if and only if $\alpha_{i}^{2} \leq 1$ for all $1 \leq i \leq N-1$ because, due to (62)-(64), $\mathbf{S}^{-1} \geq 0$ if and only if $\alpha_{i}^{2} \leq 1$. If for some $k$ we have $\alpha_{k}^{2}>1$, then the solution to the optimization problem (79)-(83) does not exist at all. This is because $\alpha_{k}^{2}$ cannot be lower than the square of (86), or else conditions (81) and (82) would not hold.

The minimum total boundary energy factorization (49) of a positive-definite covariance matrix $\mathbf{J}$ (where $\mathbf{J}^{-1}$ is tridiagonal) into positive-semidefinite factors $\mathbf{W}_{f}, \mathbf{W}_{b}$, and $\mathbf{S}$ is given by the following algorithm:

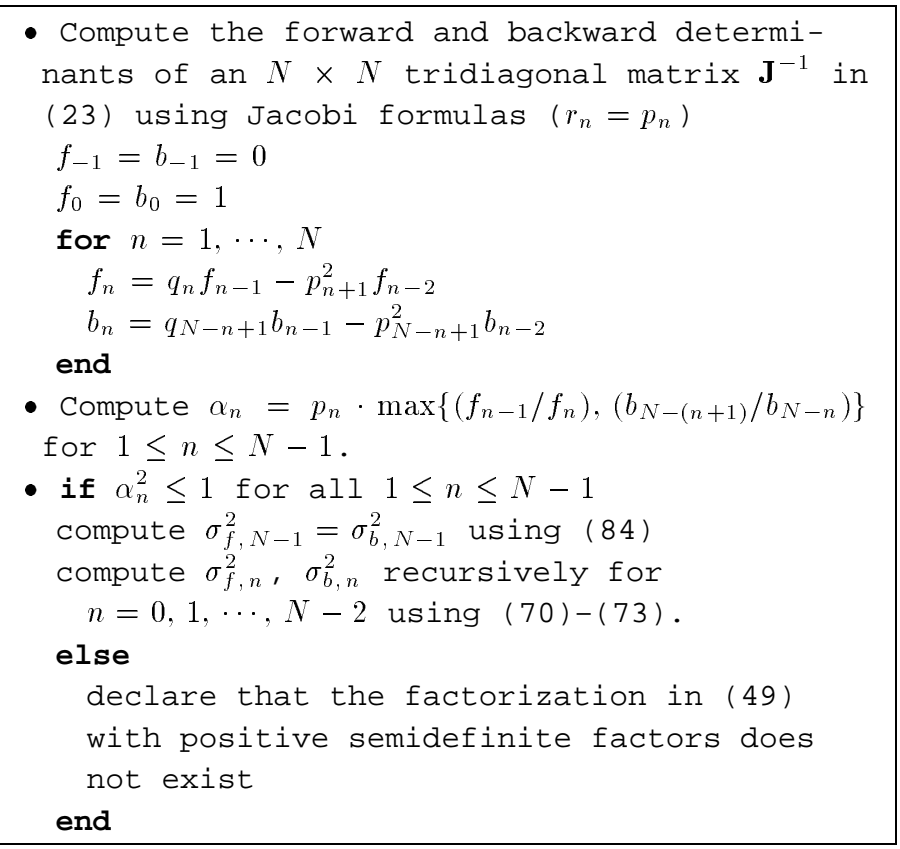

In some cases (when $p_{n}$ are of the same sign for all $1 \leq n \leq$ $N-1$ ), we may impose a further restriction on the total error energy minimization problem, namely, that the essential component factor $\mathbf{S}$ in (49) be Toeplitz. ${ }^{4}$ This factorization is interesting because we can then say that the essential component $x_{n}$ is the best wide-sense stationary process fit (in the minimum boundary energy sense) to the process whose potential matrix is $\mathbf{J}^{-1}$. The solution to this optimization problem exists whenever the solution to the original optimization problem (79)-(83) exists. Since the matrix $\mathbf{S}$ is required to be Toeplitz, the off-diagonal element in $\mathbf{S}$ that satisfies positive semidefiniteness of all three factors $\mathbf{W}_{f}, \mathbf{W}_{b}$, and $\mathbf{S}$ is given by that $\alpha_{n}$ in (86) with the largest magnitude, i.e.,

$$
\alpha=\operatorname{sign}\left(p_{1}\right) \max _{1 \leq n \leq N-1}\left\{\left|p_{n}\right| \cdot \max \left\{\frac{f_{n-1}}{f_{n}}, \frac{b_{N-(n+1)}}{b_{N-n}}\right\}\right\}
$$

where we assumed that $p_{1}$ is of the same sign as all other $p_{n}$, $1 \leq n \leq N-1$. The forward and backward independent-

\footnotetext{
${ }^{4} \mathrm{~A}$ matrix $\mathbf{A}$ is Toeplitz if $A(i, j)=A(i+1, j+1)$ for any $i, j$.
} 
increment process variances are then obtained by recursively solving (70)-(73).

\section{B. Examples}

We now consider several important special cases.

\section{Example 1 (DST-Diagonalized Matrix):}

We assume next that the matrix $\mathbf{J}^{-1}$ in (23) is Toeplitz positive-definite, where $q_{n}=q$ and $p_{n}=r_{n}=p$

$$
\mathbf{J}^{-1}=\left[\begin{array}{ccccc}
q & p & & & 0 \\
p & q & p & & \\
& q & \ddots & \ddots & \\
& & \ddots & q & p \\
\mathbf{0} & & & p & q
\end{array}\right] .
$$

This matrix is diagonalized by the discrete sine transform (DST) [5].

We are looking to find a factorization like (53) of a process $z_{n}$ whose covariance matrix is $\mathbf{J}$, and whose essential component $x_{n}$ has a Toeplitz covariance matrix $\mathbf{S}$, i.e., the $\left\{\alpha_{i}\right\}$ in (49) are all equal to $\alpha$ and given by (87), and whose total boundary error energy (69) is minimized.

The solution in (84) gives

$$
\sigma_{f, N-1}^{2}=\sigma_{b, N-1}^{2}=\left[\left(\frac{\alpha}{p}\right)^{N-1} \frac{b_{N-1} f_{N-1}}{f_{N}}\right]^{\frac{1}{2}} \text {. }
$$

For the closed-form solution for $f_{i}$, see Appendix B. It is now simple to determine $\alpha$ using the following lemma.

Lemma 3: For a symmetric Toeplitz-positive positive-definite matrix $\mathbf{J}^{-1}$ in (88), the following inequality holds for all integers $i \geq 1$ :

$$
\frac{f_{i}}{f_{i+1}} \geq \frac{f_{i-1}}{f_{i}}
$$

Proof of Lemma 3: by induction. Since $\mathbf{J}^{-1} \geq 0$, utilizing Jacobi recursions, we have $f_{i}=q f_{i-1}-p^{2} f_{i-2} \geq 0$ for all $i \geq 2$.

Initial Step:

$$
\begin{aligned}
f_{0} & =1 \\
f_{1} & =q \geq 0 \text { since } \mathbf{J}^{-1} \geq 0 \\
f_{2} & =q^{2}-p^{2} \geq 0 \text { since } \mathbf{J}^{-1} \geq 0 \\
\Longrightarrow \frac{f_{1}}{f_{2}} & =\frac{q}{q^{2}-p^{2}} \geq \frac{1}{q}=\frac{f_{0}}{f_{1}} .
\end{aligned}
$$

Inductive Step:

$$
\begin{aligned}
& \frac{f_{i-1}}{f_{i}} \geq \frac{f_{i-2}}{f_{i-1}} \\
\Longrightarrow & q f_{i} f_{i-1}-p^{2} f_{i} f_{i-2} \geq q f_{i} f_{i-1}-p^{2} f_{i-1}^{2} \\
\Longrightarrow & f_{i} \underbrace{\left(q f_{i-1}-p^{2} f_{i-2}\right)}_{f_{i}} \geq f_{i-1} \underbrace{\left(q f_{i}-p^{2} f_{i-1}\right)}_{f_{i+1}} \\
\Longrightarrow & \frac{f_{i}}{f_{i+1}} \geq \frac{f_{i-1}}{f_{i}} .
\end{aligned}
$$

Combining Lemma 3 with (87), we get

$$
\frac{\alpha}{p} \geq \frac{f_{N-2}}{f_{N-1}}
$$

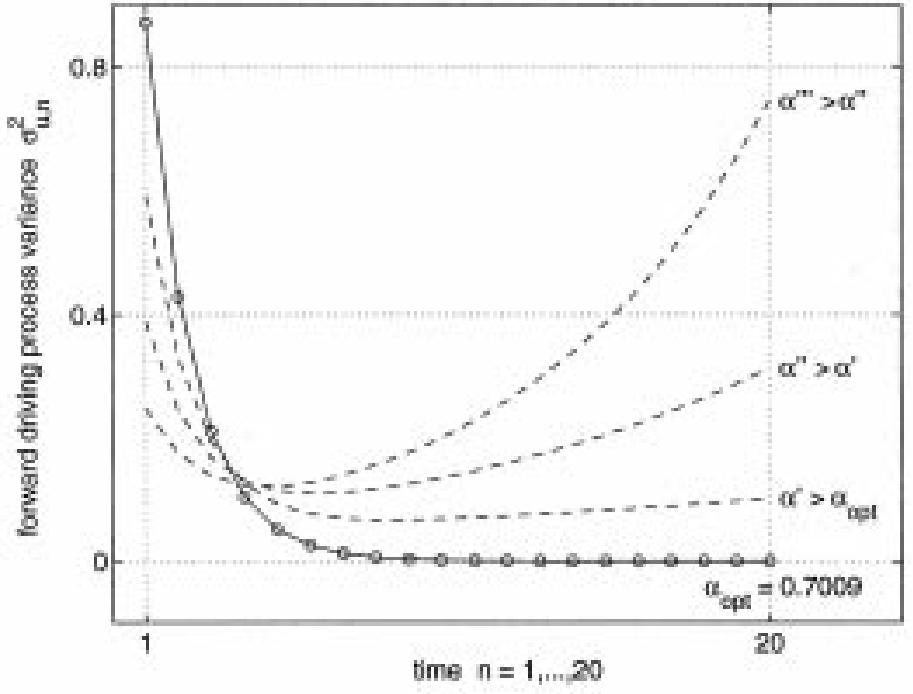

Fig. 1. Variance of the forward boundary error process $u_{n}$. For the optimal choice of $\alpha$, the error variance is maximally concentrated at the beginning of the process.

hence the Toeplitz matrix $\mathbf{S}$ that satisfies (80)-(83) and minimizes (79) has the off-diagonal element

$$
\alpha=p \frac{f_{N-2}}{f_{N-1}} \text {. }
$$

If $\alpha^{2} \leq 1$, the solution is valid, and the rest of the variables $\sigma_{f, i}^{2}$ and $\sigma_{b, i}^{2}$ needed in (49) can be obtained by substituting (89) and (91) into (70)-(73) and solving recursively.

We illustrate the properties of the solution with an example. Let $\mathbf{J}^{-1}$ be a $20 \times 20$ tridiagonal Toeplitz matrix $(N=20)$ with the elements on the main diagonal equaling $q=1$ and the elements on the first upper and lower diagonals equaling $p=0.47$. The solution that minimizes the total boundary error energy is $\alpha_{\mathrm{opt}}=0.7009$ and $\sigma_{f, N-1}^{2}=\sigma_{b, N-1}^{2}=1.7120$. The variance of the forward boundary error process $u_{n}$ that minimizes the total boundary error energy is plotted in Fig. 1 along with several other variances of the boundary error process for suboptimal factorizations (53). We observe from Fig. 1 that not only is the total forward error energy $\sum_{n=0}^{N-1} \sigma_{u, n}^{2}=\sigma_{f, N-1}^{2}$ minimized, but also the variance terms $\sigma_{u, n}^{2}$ are mostly concentrated at the beginning of the process, i.e., closest to the beginning boundary, and die as we move away from the boundary. Similar conclusions hold for the backward boundary error process for the optimal factorization, whose variance dies out as we move away in the other direction.

\section{Example 2 (DCT-Diagonalized Matrix):}

We assume next that the matrix $\mathbf{J}^{-1}$ in (23) is positive-definite and has the form

$$
\mathbf{J}^{-1}=\left[\begin{array}{ccccc}
1+p & p & & & \mathbf{0} \\
p & 1 & p & & \\
& 1 & \ddots & \ddots & \\
& & \ddots & 1 & p \\
\mathbf{0} & & & p & 1+p
\end{array}\right]
$$

where clearly $q_{1}=q_{N}=1+p, q_{n}=q=1$ for $2 \leq n \leq N-1$, and $r_{n}=p_{n}=p$. This matrix is diagonalized by the discrete cosine transform (DCT) [5]. 


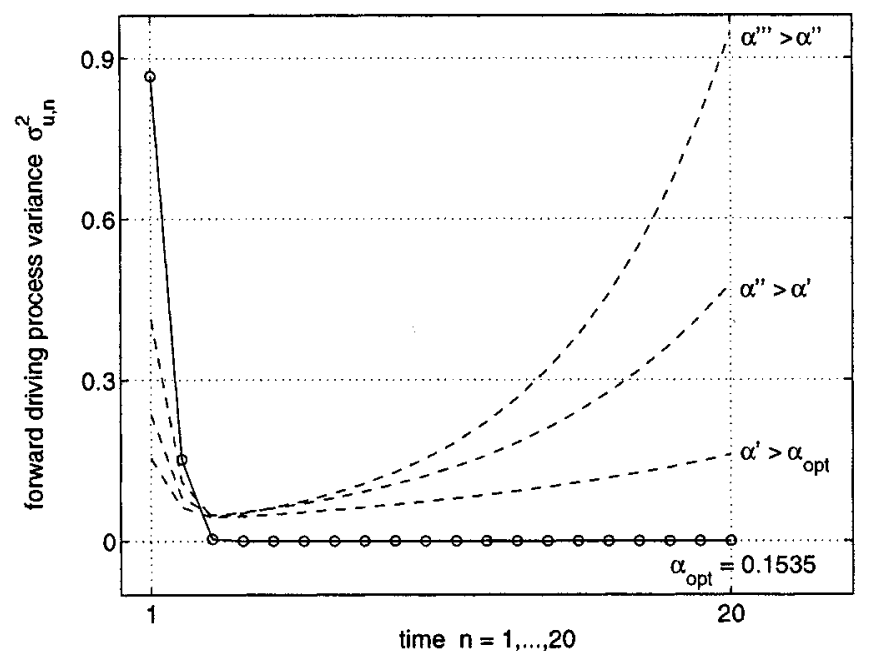

Fig. 2. Variance of the forward boundary error process $u_{n}$ for $p=0.15$ For the optimal choice of $\alpha$, the error variance is maximally concentrated at the beginning of the process.

We look for a factorization like (53) of a process $z_{n}$ whose covariance matrix is $\mathbf{J}$, and whose essential component $x_{n}$ has a Toeplitz covariance matrix $\mathbf{S}$, and whose total boundary error energy (69) is minimized.

Similarly to proving Lemma 3, we can show by an inductive argument that

$$
\frac{f_{i}}{f_{i+1}} \stackrel{p>0}{\gtrless} \underset{p<0}{\gtrless} \frac{f_{i-1}}{f_{i}}, \quad \text { for } 0 \leq i \leq N-2
$$

i.e., for $p>0$, the sequence $\left\{f_{i} / f_{i+1}\right\}$ is monotonically increasing, and for $p<0$ it is monotonically decreasing. Combining this with (87), we get that the Toeplitz matrix $\mathbf{S}$ that satisfies (80)-(83) and minimizes (79) has the off-diagonal element

$$
\alpha= \begin{cases}p \frac{f_{N-2}}{f_{N-1}}, & \text { for } p>0 \\ p \frac{f_{0}}{f_{1}}=\frac{p}{1+p}, & \text { for } p<0 .\end{cases}
$$

If $\alpha^{2} \leq 1$, the solution is valid, and the remaining variables $\sigma_{f, i}^{2}$ and $\sigma_{b, i}^{2}$ needed in (49) can be obtained by substituting (89) and (91) into (70)-(73) and solving recursively.

In Figs. 2 and 3, optimization results are plotted for $p=$ 0.15 and $p=-0.15$, respectively. The plots show the variance of the process $u_{n}$ driving the forward independent-increment process $w_{f, n}$. Both plots reveal that, for the optimally chosen $\alpha$, the boundary error is minimized. At $n=1$ (beginning of the process), the variance is largest for the optimal process. Likewise, at $n=20$ (end of the process), the variance is smallest for the optimal process. This is revealed by the plots of the variance for suboptimal choices $\alpha^{\prime}, \alpha^{\prime \prime}$, and $\alpha^{\prime \prime \prime}$ in Figs. 2 and 3.

\section{GAUSS-MARKOV APPROXIMATIONS AND INFORMATION LOSS}

In many engineering applications, it is desirable to approximate a discrete-time Gaussian random process by a GaussMarkov random process of order $L$. Since the inverse covariance matrix (the potential matrix) of an $L$ th-order Gauss-Markov

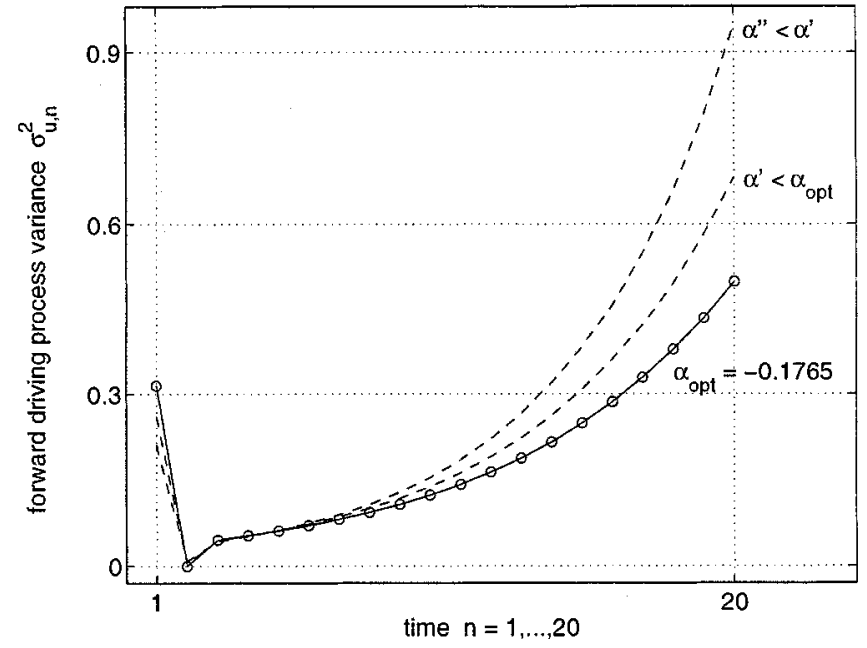

Fig. 3. Variance of the forward boundary error process $u_{n}$ for $p=-0.15$.

process has an $L$-banded structure [3], Gauss-Markov approximations lead to computationally efficient algorithms, see, e.g., [6]-[8]. We address here the following question. Given a Gaussian random process and an order $L \geq 0$, what is the Gauss-Markov random process of order $L$ that "best" fits, in some sense, the observed Gaussian process? We consider two optimality criteria, the Kullback-Leibler mean information (KLMI) and the entropy. The formulations of the problems in these two cases are different, but, as we shall see, they lead to the same solution.

\section{A. Optimal Gauss-Markov Approximation}

Theorem 3 (Maximum Entropy Covariance Extension): Let C be an arbitrary non-Toeplitz covariance matrix. Let $\dot{Z}_{N}^{1}$ be a zero-mean random vector, such that the $L$-band of its covariance matrix $\mathbf{R}$ equals the $L$-band of $\mathbf{C}$. The entropy of $\dot{Z}_{N}^{1}$ is maximized when $\dot{Z}_{N}^{1}$ is an $L$ th-order Gauss-Markov vector whose covariance matrix $\mathbf{R}$ is the $L$-band extension of $\mathbf{C}$ given by (7).

Proof: This theorem is a special case of the general theorem that establishes the following results: i) a multivariate Gaussian probability density function (pdf) maximizes the entropy under the given $L$-band covariance constraint, ii) the inverse of the covariance matrix is $L$-banded, and iii) the $L$-band of $\mathbf{R}$ equals the $L$-band of $\mathbf{C}$. The proof of part i) is well known and can be found in [9]. Parts ii) and iii) have been independently proved in [10] and [11]. In [12], the theorem is presented in one piece. Combining results i)-iii) with the Decomposition Theorem (Theorem 2), we have that the $L$-band extension as defined in (7) is the maximum-entropy covariance extension.

Theorem 4 (Kullback-Leibler Optimal Covariance): Let $\mathbf{C}$ be the covariance matrix of a zero-mean Gaussian vector $Z_{N}^{1}$. Let $\mathbf{R}$ be the covariance matrix of the $L$ th-order Gauss-Markov approximation vector $\dot{Z}_{N}^{1}$. The matrix $\mathbf{R}$ that minimizes the Kullback-Leibler mean information between $Z_{N}^{1}$ and $\dot{Z}_{N}^{1}$ is the $L$-band extension of $\mathbf{C}$ given by (7).

Proof: The proof of the general theorem can be found in [12], where it is presented as a corollary to the Triangle Equality 
Theorem for matrices with banded support. Application of Theorem 2 proves our special case.

Theorems 3 and 4 both lead to the $L$-band extension as the solution to their respective optimizations, but the two optimization criteria are not the same. In Theorem 3, the matrix $\mathbf{C}$ is not assumed to be a covariance of a Gaussian vector and the optimization is done over all possible probability density functions. Contrary to that, in Theorem 4, the matrix $\mathbf{C}$ is assumed to be the covariance matrix of a Gaussian random vector and the optimal solution is sought only in the space of Gauss-Markov processes. However, these two criteria are equivalent when $\mathbf{C}$ is assumed to be the covariance matrix of a Gaussian vector and the solution is confined to Gauss-Markov processes, which is formally established by the Dual Optimization Lemma [12].

\section{B. Autoregressive Process Interpretation}

Suppose $\mathbf{R}$ is the $L$-band extension of a covariance matrix $\mathbf{C}$. Since $\mathbf{R}$ is a symmetric positive-definite matrix, from Section II-C we have that the UDL decomposition of $\mathbf{R}^{-1}$ (we also refer to it as the upper Cholesky decomposition) is

$$
\mathbf{R}^{-1}=\mathbf{U}_{\mathbf{R}} \mathbf{D}_{\mathbf{R}} \mathbf{U}_{\mathbf{R}}^{T}
$$

where

$$
\mathbf{D}_{\mathbf{R}}=\operatorname{diag}\left[\left(1 / \sigma_{1}^{2}\right), \cdots,\left(1 / \sigma_{N}^{2}\right)\right]
$$

has positive diagonal entries. The elements of $\mathbf{U}_{\mathbf{R}}$ and the (inverses of the) diagonal elements, $\delta_{k}=\sigma_{k}^{2}$, are given by (18) and (20). When $\mathbf{C}$ is a symmetric positive-definite matrix, we recognize (18) and (20) as solutions to "nonstationary" Yule-Walker equations (normal equations), also provided in [12]. Similar to (95), the upper Cholesky decomposition of $\mathbf{C}^{-1}$ is

$$
\mathbf{C}^{-1}=\mathbf{V}_{\mathbf{C}} \boldsymbol{\Lambda}_{\mathbf{C}} \mathbf{V}_{\mathbf{C}}^{T}
$$

where

$$
\mathbf{\Lambda}_{\mathbf{C}}=\operatorname{diag}\left[\left(1 / \lambda_{1}^{2}\right), \cdots,\left(1 / \lambda_{N}^{2}\right)\right] .
$$

Clearly, since the first $(L+1) \times(L+1)$ principal minor of $\mathbf{R}$ and $\mathbf{C}$ are identical, we have that $\sigma_{k}^{2}=\lambda_{k}^{2}$ for $k \leq L+1$. For the same reason, the first $L+1$ columns of $\mathbf{V}_{\mathbf{C}}$ and $\mathbf{U}_{\mathbf{R}}$ are identical.

If $\mathbf{C}$ is the covariance matrix of a Gaussian vector $\underline{z}_{N}^{1}$, then the $L$-band extension $\mathbf{R}$ is the covariance matrix of the optimal (in the maximum-entropy sense) $L$ th-order Gauss-Markov approximation vector $\dot{\dot{z}}_{N}^{1}$. The upper Cholesky decompositions of $\mathbf{C}^{-1}$ and $\mathbf{R}^{-1}$ are canonic forms for representing the time-varying causal autoregressive filters that generate the processes $\underline{z}_{N}^{1}$ and $\underline{\dot{z}}_{N}^{1}$ by filtering a zero-mean unit-variance white Gaussian process $w_{k}$. This is shown in Fig. 4. In Fig. 4, the filter of Fig. 4(a) creates the original vector $\underline{z}_{N}^{1}$ while the filter of Fig. 4(b) creates $\underline{\dot{z}}_{N}^{1}$. The number of tap-weights of filter (a) grows linearly with every time instant. Filter (b) is identical to filter (a) up to (and including) the time instant $k=L+1$. For all time instants $k>L$ the number of tap weights of filter (b) is $L$. Hence, the two filters differ for $k>L+1$.

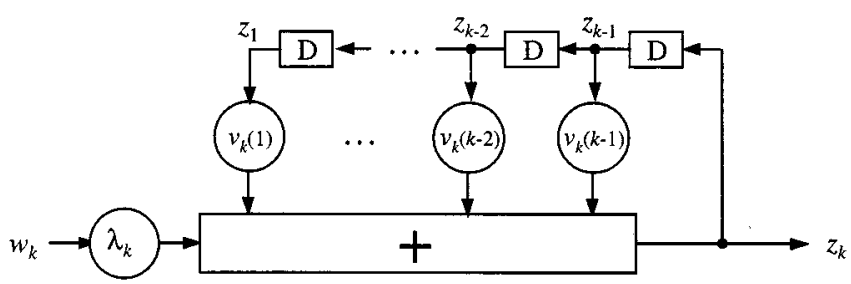

(a)

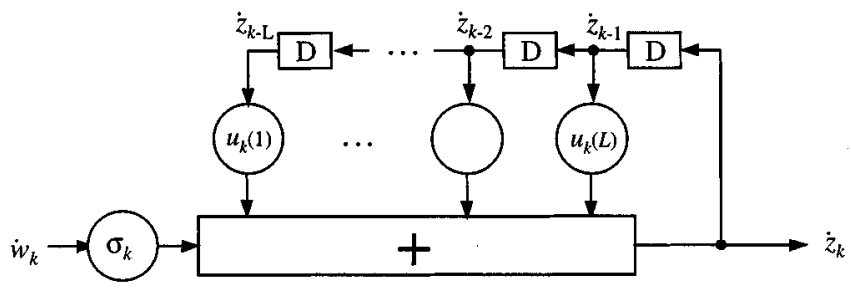

(b)

Fig. 4. Autoregressive filters creating (a) the Gaussian process $z_{k}$ and (b) its $L$ th-order Gauss-Markov approximation $\dot{z}_{k}$.

\section{Minimal Information Loss}

We define the information loss as the Kullback-Leibler distance between the random vector $\underline{z}_{N}^{1}$ and its $L$ th-order approximation $\underline{\dot{z}}_{N}^{1}$. If $\mathbf{C}$ is the covariance matrix of a Gaussian vector $\underline{z}_{N}^{1}$ and $\mathbf{R}$ is the covariance matrix of the Gaussian approximation vector $\underline{\dot{z}}_{N}^{1}$, the information loss (Kullback-Leibler distance) of the approximation is

$$
I(N)=\frac{1}{2} \ln \frac{\operatorname{det} \mathbf{R}}{\operatorname{det} \mathbf{C}}+\frac{1}{2} \operatorname{tr}\left(\mathbf{C R}^{-1}\right)-\frac{N}{2} .
$$

If $\mathbf{R}$ is the $L$-band extension of $\mathbf{R}$, then $\underline{\dot{x}}_{N}^{1}$ is the optimal $L$ th-order Gauss-Markov approximation. Due to Theorem 4, the information loss is then minimized. Invoking Corollaries 1.1 and 1.2 and the upper Cholesky decompositions (95) and (96), we get that the minimal information loss is

$$
I(N)=\frac{1}{2} \ln \prod_{k=L+2}^{N} \frac{\sigma_{k}^{2}}{\lambda_{k}^{2}}
$$

i.e., the minimal information loss equals the difference of the entropies of the two uncorrelated Gaussian random sequences $\left\{\sigma_{k} \dot{w}_{k}\right\}$ and $\left\{\lambda_{k} w_{k}\right\}$ that drive the two autoregressive filters (a) and (b) in Fig. 4, respectively.

It is interesting to contrast the approximation based on the low-order $L$-band extension and the approximation based on the low-rank covariance approximation (Karhunen-Loève decomposition). Table I contrasts these decompositions. In the last row in Table I we characterize the Karhunen-Loève approximation as "static," while we view the band extension as "dynamic." This characterization is justified by the typical signal processing scenarios where the two approximations are used. Typical applications of the low-rank approximation are suboptimal one-shot detection/estimation problems such as the radar detection problem [13]. Typical usages of the approximations based on low-band extensions are in suboptimal dynamic detection/estimation applications such as the Viterbi algorithm [6], or the Kalman-Bucy filter [7]. 
TABLE I

CONTRASTING THE KARHUNEN-LOÈVE LOW-RANK APPROXIMATION WITH THE LOW-BANDWIDTH APPROXIMATION

\begin{tabular}{|c|c|c|}
\hline & $\begin{array}{c}\text { Karhunen-Loéve } \\
\text { approximation }\end{array}$ & $\begin{array}{c}\text { Low bandwidth } \\
\text { approximation }\end{array}$ \\
\hline \hline matrix decomposition & $\begin{array}{c}\text { singular value decomp. } \\
\mathbf{C}=\mathbf{Q}_{\mathbf{C}} \mathbf{\Sigma}_{\mathbf{C}} \mathbf{Q}_{\mathbf{C}}^{\mathrm{T}}\end{array}$ & $\begin{array}{c}\text { Cholesky decomp. } \\
\mathbf{C}^{-1}=\mathbf{V}_{\mathbf{C}} \boldsymbol{\Lambda}_{\mathbf{C}} \mathbf{V}_{\mathbf{C}}^{\mathrm{T}}\end{array}$ \\
\hline approximation constraint & reduces rank $r$ & reduced bandwidth $L$ \\
\hline distance measure & $\begin{array}{c}\text { Froebenius-norm distance } \\
\left.\text { (energy loss } e^{2}\right)\end{array}$ & $\begin{array}{c}\text { Kullback-Leibler distance } \\
\text { (information loss } I)\end{array}$ \\
\hline decomposition of the & $\mathbf{R}=\mathbf{P}_{\mathbf{R}} \mathbf{S}_{\mathbf{R}} \mathbf{P}_{\mathbf{R}}^{\mathrm{T}}$ & $\mathbf{R}^{-1}=\mathbf{U}_{\mathbf{R}} \mathbf{D}_{\mathbf{R}} \mathbf{U}_{\mathbf{R}}^{\mathrm{T}}$ \\
\hline approximation & $e^{2}=\operatorname{tr}\left(\mathbf{\Sigma}_{\mathbf{C}}\right)-\operatorname{tr}\left(\mathbf{S}_{\mathbf{R}}\right)$ & $I=\ln \left(\right.$ det $\left.\boldsymbol{\Lambda}_{\mathbf{C}}\right)-\ln \left(\operatorname{det} \mathbf{D}_{\mathbf{R}}\right)$ \\
\hline quality of optimal approximation & static & dynamic \\
\hline character & & \\
\hline
\end{tabular}

\section{SUMMARY}

This paper has presented a novel nested algorithm to invert general matrices whose inverses are known to be banded. This algorithm uses only the entries of the (full) matrix corresponding to the nonzero entries of its inverse. We apply the nested algorithm to obtain three important results: explicit inverses for tridiagonal matrices; a novel factorization of first-order noncausal Gauss-Markov processes; and approximations of Gauss processes by Gauss-Markov processes and the corresponding information loss.

We believe that our inverses of general tridiagonal matrices are the first time such explicit inverses are available. These expressions when applied to certain matrices provide the closed-form expressions for the covariances of GMrp's whose Karhunen-Loève transforms are the discrete cosine transform (DCT) and the discrete sine transform (DST).

The inverses of tridiagonal matrices are given as the element-wise (or Hadamard) product of three matrices. When these are covariance matrices in their own right, they provide an interesting and novel interpretation for noncausal first-order Gauss-Markov random processes. The noncausal GMrp is factored as the product of three independent-increment processes: a forward process that takes care of the initial condition of the GMrp; a backward process that accounts for the terminal condition of the GMrp; and a variance stationary (free at the boundaries) GMrp. This canonical decomposition of a GMrp as a factorization of independent processes is striking in its own right, being very different from the usual decomposition of processes as sums of elementary processes, or autoregressive type representations.

Finally, we apply the nested algorithm to the problem of approximating Gaussian processes by noncausal GMrp's and compute explicitly the information loss of the approximation.

\section{APPENDIX A \\ PROOFS}

Proof of Lemma 1: We recall the matrix-inversion lemma (see e.g., [14]) for block-partitioned matrices. Assuming $\mathbf{A}^{-1}$ exists

$$
\left[\begin{array}{cc}
\mathbf{A} & \mathbf{B} \\
\mathbf{C}^{T} & \mathrm{D}
\end{array}\right]^{-1}=\left[\begin{array}{cc}
\mathbf{A}^{-1}+\mathbf{E} \boldsymbol{\Delta}^{-1} \mathbf{F} & -\mathbf{E} \boldsymbol{\Delta}^{-1} \\
-\boldsymbol{\Delta}^{-1} \mathbf{F} & \boldsymbol{\Delta}^{-1}
\end{array}\right]
$$

where

$$
\begin{aligned}
& \boldsymbol{\Delta}=\mathbf{D}-\mathbf{C}^{T} \mathbf{A}^{-1} \mathbf{B} \\
& \mathbf{E}=\mathbf{A}^{-1} \mathbf{B} \\
& \mathbf{F}=\mathbf{C}^{T} \mathbf{A}^{-1} .
\end{aligned}
$$

We now prove Lemma 1. Partition the matrices $\mathbf{R}_{N}^{1}$ and $\mathbf{R}_{N}^{N-L}$ as follows:

$$
\begin{aligned}
\mathbf{R}_{N}^{1} & =\left[\begin{array}{cc}
\mathbf{R}_{N-1}^{1} & \underline{B} \\
\underline{C}^{T} & r
\end{array}\right] \\
\mathbf{R}_{N}^{N-L} & =\left[\begin{array}{cc}
\mathbf{R}_{N-1}^{N-L} & \underline{b} \\
\underline{c}^{T} & r
\end{array}\right] .
\end{aligned}
$$

With the MatLab notation

$$
\begin{aligned}
\underline{B} & =\mathbf{R}_{N}^{1}(1: N-1, N) \\
\underline{C}^{T} & =\mathbf{R}_{N}^{1}(N, 1: N-1)
\end{aligned}
$$

and likewise for $\underline{b}$ and $\underline{c}^{T}$. Applying the partitioned matrix inverse theorem (99) to (100), we get

$$
\begin{aligned}
& {\left[\mathbf{R}_{N}^{1}\right]^{-1}-\left[\begin{array}{cc}
{\left[\mathbf{R}_{N-1}^{1}\right]^{-1}} & \underline{0} \\
\underline{0}^{T} & 0
\end{array}\right]} \\
& \quad=\frac{1}{H}\left[\begin{array}{cc}
{\left[\mathbf{R}_{N-1}^{1}\right]^{-1} \underline{B} \underline{C}^{T}\left[\mathbf{R}_{N-1}^{1}\right]^{-1}} & -\left[\mathbf{R}_{N-1}^{1}\right]^{-1} \underline{B} \\
-\underline{C}^{T}\left[\mathbf{R}_{N-1}^{1}\right]^{-1} & 1
\end{array}\right]
\end{aligned}
$$

and

$$
\begin{aligned}
& {\left[\mathbf{R}_{N}^{N-L}\right]^{-1}-\left[\begin{array}{cc}
{\left[\mathbf{R}_{N-1}^{N-L}\right]^{-1}} & \underline{0} \\
\underline{0}^{T} & 0
\end{array}\right]} \\
& \quad=\frac{1}{h}\left[\begin{array}{cc}
{\left[\mathbf{R}_{N-1}^{N-L}\right]^{-1} \underline{b} \underline{c}^{T}\left[\mathbf{R}_{N-1}^{N-L}\right]^{-1}} & -\left[\mathbf{R}_{N-1}^{N-L}\right]^{-1} \underline{b} \\
-\underline{c}^{T}\left[\mathbf{R}_{N-1}^{N-L}\right]^{-1} & 1
\end{array}\right]
\end{aligned}
$$

where

and

$$
H=r-\underline{C^{T}}\left[\mathbf{R}_{N-1}^{1}\right]^{-1} \underline{B}
$$

$$
h=r-\underline{c}^{T}\left[\mathbf{R}_{N-1}^{N-L}\right]^{-1} \underline{b}
$$


are the respective Schur complements of $r$ in (100), see, e.g., [14]. Notice that (101) and (102) combine to give (6) if the following holds:

$$
\begin{aligned}
{\left[\mathbf{R}_{N-1}^{1}\right]^{-1} \underline{B} } & =\left[\begin{array}{c}
\underline{0} \\
{\left[\mathbf{R}_{N-1}^{N-L}\right]^{-1} \underline{b}}
\end{array}\right] \\
\underline{C}^{T}\left[\mathbf{R}_{N-1}^{1}\right]^{-1} & =\left[\begin{array}{ll}
\underline{0}^{T} & \underline{c}^{T}\left[\mathbf{R}_{N-1}^{N-L}\right]^{-1}
\end{array}\right] .
\end{aligned}
$$

We now prove (104) and (105). Partition the matrix $\mathbf{R}_{N-1}^{1}$ as

$$
\mathbf{R}_{N-1}^{1}=\left[\begin{array}{cc}
\mathbf{X} & \mathbf{Y} \\
\mathbf{Z} & \mathbf{R}_{N-1}^{N-L}
\end{array}\right]
$$

Applying the partitioned matrix inverse theorem (see e.g., [14]) on (106), we get

$$
\left[\mathbf{R}_{N-1}^{1}\right]^{-1}=\left[\begin{array}{cc}
\mathbf{0} & \mathbf{0} \\
\mathbf{0} & {\left[\mathbf{R}_{N-1}^{N-L}\right]^{-1}}
\end{array}\right]+\left[\begin{array}{c}
\mathbf{I} \\
\mathbf{F}
\end{array}\right] \mathbf{S}^{-1}\left[\begin{array}{ll}
\mathbf{I} & \mathbf{G}
\end{array}\right]
$$

where

$$
\begin{aligned}
& \mathbf{F}=-\left[\mathbf{R}_{N-1}^{N-L}\right]^{-1} \mathbf{Z} \\
& \mathbf{G}=-\mathbf{Y}\left[\mathbf{R}_{N-1}^{N-L}\right]^{-1}
\end{aligned}
$$

and

$$
\mathbf{S}=\mathbf{X}-\mathbf{Y}\left[\mathbf{R}_{N-1}^{N-L}\right]^{-1} \mathbf{Z}
$$

Since $\mathbf{R}_{N}^{N-L}$ is a principal submatrix of $\mathbf{R}_{N}^{1}$, we have that $\underline{b}$ is a subvector of $\underline{B}$, see (100), i.e., we can write

$$
\underline{B}=\left[\frac{x}{\underline{b}}\right] \text {. }
$$

Multiplying (107) by (108) from the right, we get

$$
\left[\mathbf{R}_{N-1}^{1}\right]^{-1} \underline{B}=\left[\begin{array}{c}
\underline{0} \\
{\left[\mathbf{R}_{N-1}^{N-L}\right]^{-1} \underline{b}}
\end{array}\right]+\left[\begin{array}{c}
\mathbf{S}^{-1}(\underline{x}+\mathbf{G} \underline{b}) \\
\mathbf{F S}^{-1}(\underline{x}+\mathbf{G} \underline{b})
\end{array}\right] .
$$

Since $\left[\mathbf{R}_{N}^{1}\right]^{-1}$ is $L$-banded by assumption, the last ( $N$ th) column of the left-hand side of (101) has zeros in the first $N-L-1$ entries. Hence, the same must hold for the right-hand side of (101). It follows then that the column vector $\left[\mathbf{R}_{N-1}^{1}\right]^{-1} B$ has zeros in its first $N-L-1$ entries. Since $\left[\mathbf{R}_{N-1}^{1}\right]^{-1} \underline{B}$ has zeros in its first $N-L-1$ entries, it follows from (109) that $\mathbf{S}^{-1}(\underline{x}+\mathbf{G} \underline{b})=\underline{0}$. Then we also have $\mathbf{F S}^{-1}(\underline{x}+\mathbf{G} \underline{b})=\underline{0}$. Thus (109) actually reads as (104). The proof of (105) follows from similar arguments.

Finally, we prove $H=h$.

$$
\begin{aligned}
H & =r-\underline{C}^{T}\left[\mathbf{R}_{N-1}^{1}\right]^{-1}\left[\mathbf{R}_{N-1}^{1}\right]\left[\mathbf{R}_{N-1}^{1}\right]^{-1} \underline{B} \\
& =r-\left[\underline{0}^{T} \underline{c}^{T}\left[\mathbf{R}_{N-1}^{N-L}\right]^{-1}\right]\left[\begin{array}{cc}
\mathbf{X} & \mathbf{Y} \\
\mathbf{Z} & \mathbf{R}_{N-L}^{N-L}
\end{array}\right]\left[\begin{array}{c}
\underline{0} \\
{\left[\mathbf{R}_{N-1}^{N-L}\right]^{-1} \underline{b}}
\end{array}\right] .
\end{aligned}
$$

Multiplying out the matrices on the right-hand side readily shows that $H=h$.

Proof of Lemma 2: In the proof of Lemma 1, we established that the first $N-L-1$ elements of the vectors $\left[\mathbf{R}_{N-1}^{1}\right]^{-1} \underline{B}$ and $\underline{C}^{T}\left[\mathbf{R}_{N}^{1}\right]^{-1}$ are zeros. Hence, only the lowest $(L+1) \times(L+1)$ principal submatrix of the right-hand side of (101) is nonzero. Thus the matrix on the right-hand side of (101) is $L$-banded. Since $\left[\mathbf{R}_{N}^{1}\right]^{-1}$ is also $L$-banded, it follows from (101) that $\left[\mathbf{R}_{N-1}^{1}\right]^{-1}$ is $L$-banded too because it is obtained by adding two $L$-banded matrices. With similar arguments, we can show that $\left[\mathbf{R}_{N}^{2}\right]^{-1}$ is also $L$-banded. Carrying on these arguments for smaller principal submatrices, we conclude that every principal submatrix $\mathbf{R}_{j}^{i}$ of size $(L+1) \times(L+1)$ or greater must have an $L$-banded inverse.

\section{APPENDIX B}

\section{FREQUENTLY ENCOUNTERED SYMMETRIC TRIDIAGONAL MATRICES}

We consider four examples of tridiagonal matrices that are useful in applications. The first is a well-known example and we include it just for completeness.

First-Order Stationary Gauss-Markov Process: Perhaps the only previously known nontrivial example of a tridiagonal matrix whose inverse is known is the matrix

$$
\begin{aligned}
\mathbf{J}^{-1}= & \frac{1}{1-\alpha^{2}} \\
& \cdot \underbrace{\left[\begin{array}{ccccc}
1 & -\alpha & -\alpha & 0 \\
-\alpha & 1+\alpha^{2} & -\alpha & \\
& -\alpha & 1+\alpha^{2} & -\alpha & \\
\mathbf{0} & & -\alpha & 1+\alpha^{2} & -\alpha \\
& & -\alpha & 1+\alpha^{2}
\end{array}\right]}_{\mathbf{J}_{1}^{-1}}
\end{aligned}
$$

whose inverse is the covariance matrix of a first-order stationary causal Gauss-Markov process

$$
\mathbf{J}=\left[\begin{array}{ccccc}
1 & \alpha & \alpha^{2} & \cdots & \alpha^{N-1} \\
\alpha & 1 & \alpha & \alpha^{2} & \vdots \\
\alpha^{2} & \alpha & 1 & \alpha & \alpha^{2} \\
\vdots & \alpha^{2} & \alpha & 1 & \alpha \\
\alpha^{N-1} & \cdots & \alpha^{2} & \alpha & 1
\end{array}\right]
$$

Matrix $\mathbf{J}$ is

$$
\left(1-\alpha^{2}\right) \times \mathbf{J}_{1}
$$

To find $\mathbf{J}_{1}$, we compute each of the factors in the Hadamard product formula (48). For the first two factors, and using the Jacobi determinant relations (39) and (40), we get that all determinants $f_{n}=b_{n}=1,0 \leq n \leq N-1$, and $f_{N}=b_{N}=1-\alpha^{2}$. Finally, the third factor in (48) is exactly matrix (111). So, $\mathbf{J}_{1}$ is $1 /\left(1-\alpha^{2}\right)$ times the matrix in (111), which once replaced in (112) gives that $\mathbf{J}$ is as in (111). The UDL decomposition for this example is similar in form to the decomposition in (62)-(64) with $\alpha_{i}=-\alpha$.

\section{Discrete Sine Transform (DST):}

The following symmetric Toeplitz tridiagonal matrix $\mathbf{J}^{-1}$ is diagonalized by the discrete sine transform (DST) [5]. Define the $N \times N$ matrix $\mathbf{J}^{-1}$ as

$$
\mathbf{J}^{-1}=\left[\begin{array}{ccccc}
\alpha & 1 & & & \mathbf{0} \\
1 & \alpha & 1 & & \\
& 1 & \ddots & \ddots & \\
& & \ddots & \alpha & 1 \\
\mathbf{0} & & & 1 & \alpha
\end{array}\right]=\frac{1}{\lambda}\left[\begin{array}{ccccc}
1 & \lambda & & & 0 \\
\lambda & 1 & \lambda & & \\
& \lambda & \ddots & \ddots & \\
& & \ddots & 1 & \lambda \\
\mathbf{0} & & \lambda & 1
\end{array}\right]
$$

By inspection, the constants in (23) are $q_{n}=\alpha$ and $p_{n}=r_{n}=1$. The forward and backward determinants of (113) are

$$
f_{n}=b_{n}=U_{n}\left(\frac{\alpha}{2}\right)
$$


where

$$
U_{n}(x)= \begin{cases}\cos \left(n \frac{\pi}{2}\right), & \text { for } x=0 \\ \frac{\sin (n+1) \arccos x}{\sin \arccos x} & \text { for } 0<|x|<1 \\ (n+1) x^{n}, & \text { for }|x|=1 \\ \frac{\sinh (n+1) \operatorname{arccosh} x}{\sinh \operatorname{arccosh} x}, & \text { for }|x|>1\end{cases}
$$

is the $n$ th-order Chebyshev polynomial of the second kind [15]. Substituting $p_{n}, r_{n}, f_{n}$, and $b_{n}$ into (47) and (48), we get the closed-form solution for $\mathbf{J}$. This matrix is then a covariance matrix that is diagonalized by the DST. It is the covariance of a first-order noncausal Gauss-Markov process with zero Dirichlet boundary conditions, see [1].

\section{Discrete Cosine Transform (DCT):}

The following symmetric tridiagonal matrix $\mathbf{J}^{-1}$ is diagonalized by the discrete cosine transform (DCT) [5]

$$
\begin{aligned}
& \mathbf{J}^{-1}=\left[\begin{array}{ccccc}
1+\alpha & 1 & & & \mathbf{0} \\
1 & \alpha & 1 & & \\
& 1 & \ddots & \ddots & \\
& & \ddots & \alpha & 1 \\
\mathbf{0} & & & 1 & 1+\alpha
\end{array}\right] \\
& =\frac{1}{\lambda}\left[\begin{array}{ccccc}
1+\lambda & \lambda & & & \mathbf{0} \\
\lambda & 1 & \lambda & & \\
& \lambda & \ddots & \ddots & \\
& & \ddots & 1 & \lambda \\
\mathbf{0} & & & \lambda & 1+\lambda
\end{array}\right], \quad \text { for } \alpha=\frac{1}{\lambda} \text {. }
\end{aligned}
$$

By inspection, the constants in (23) are $q_{1}=q_{N}=1+\alpha$, $q_{n}=\alpha$ for $2 \leq n \leq N-1$, and $p_{n}=r_{n}=1$. The forward and backward determinants of (116) are

$$
\begin{aligned}
& f_{n}=b_{n}=U_{n}\left(\frac{\alpha}{2}\right)+U_{n-1}\left(\frac{\alpha}{2}\right), \quad \text { for } 0 \leq n \leq N-1 \\
& f_{N}=b_{N}=(1+\alpha) f_{N-1}-f_{N-2}
\end{aligned}
$$

where $U_{n}(x)$ is the $n$ th-order Chebyshev polynomial of the second kind, see (115). Substituting them into (47) and (48), we get the closed-form solution for $\mathbf{J}$. This matrix is a covariance matrix that is diagonalized by the DCT. It is the covariance of a first-order noncausal Gauss-Markov process with zero nonsymmetric Neumann boundary conditions, see [1].

\section{Symmetric Boundary Conditions Matrix:}

The following matrix $\mathbf{J}^{-1}$ is associated with a first-order Gauss-Markov (acausal) random field defined on a finite lattice with symmetric boundary conditions [3]

$$
\mathbf{J}^{-1}=\left[\begin{array}{cccccc}
\alpha & 2 & & & & 0 \\
2 & \alpha & 1 & & & \\
& 1 & \ddots & \ddots & & \\
& & \ddots & \alpha & 1 & \\
& & & 1 & \alpha & 2 \\
0 & & & & 2 & \alpha
\end{array}\right]
$$

$$
=\frac{1}{\lambda}\left[\begin{array}{cccccc}
1 & 2 \lambda & & & & 0 \\
2 \lambda & 1 & \lambda & & & \\
& \lambda & \ddots & \ddots & & \\
& & \ddots & 1 & \lambda & \\
& & & \lambda & 1 & 2 \lambda \\
0 & & & & 2 \lambda & 1
\end{array}\right], \quad \text { for } \alpha=\frac{1}{\lambda} .
$$

By inspection, the constants in (23) are $p_{1}=r_{1}=p_{N-1}=$ $r_{N-1}=2, p_{n}=r_{n}$ for $2 \leq n \leq N-2$, and $q_{n}=\alpha$. The forward and backward determinants of (119) are

$$
\begin{aligned}
& f_{0}=b_{0}=1 \\
& f_{n}=b_{n}=4 U_{n}\left(\frac{\alpha}{2}\right)-3 \alpha U_{n-1}\left(\frac{\alpha}{2}\right), \quad \text { for } 1 \leq n \leq N-1
\end{aligned}
$$

$$
f_{N}=b_{N}=\alpha f_{N-1}-4 f_{N-2}
$$

where $U_{n}(x)$ is the $n$ th-order Chebyshev polynomial of the second kind, see (115). Substituting them into (47) and (48), we get the closed-form solution for $\mathbf{J}$. This matrix is the covariance of a first-order noncausal Gauss-Markov process with zero symmetric Neumann boundary conditions, see [3], [1].

\section{REFERENCES}

[1] J. M. F. Moura and M. G. S. Bruno, "DCT/DST and Gauss-Markov fields: Conditions for equivalence," IEEE Trans. Signal Processing, vol. 46, pp. 2571-2574, Sept. 1998.

[2] G. H. Golub and C. F. Van Loan, Matrix Computations. Baltimore, MD: Johns Hopkins Univ. Press, 1983.

[3] J. M. F. Moura and N. Balram, "Recursive structure of noncausal Gauss Markov random fields," IEEE Trans. Inform. Theory, vol. 38, pp. 334-354, Mar. 1992.

[4] J. W. Woods, "Two-dimensional discrete Markovian fields," IEEE Trans. Inform. Theory, vol. IT-18, pp. 232-240, Mar. 1972.

[5] A. K. Jain, Fundamentals of Digital Image Processing. Englewood Cliffs, NJ: Prentice-Hall, 1968.

[6] A. Kavčić and J. M. F. Moura, "The Viterbi algorithm and Markov noise memory," IEEE Trans. Inform. Theory, vol. 46, pp. 291-301, Jan. 2000.

[7] A. Asif and J. M. F. Moura, "Data assimilation in large time varying multidimensional fields," IEEE Trans. Image Processing, vol. 11, pp. 1593-1607, Nov. 1999.

[8] T. M. Chin, W. C. Karl, and A. S. Willsky, "A distributed and iterative square root algorithm for space-time filtering," Automatica, vol. 31, pp. 67-82, Jan. 1995.

[9] T. M. Cover and J. A. Thomas, Elements of Information Theory. New York: Wiley, 1991.

[10] R. Grone, C. R. Johnson, E. M. Sá, and H. Wolkowicz, "Positive definite completions of partial Hermitian matrices," Linear Algebra Appl., vol. 58, pp. 109-124, 1984.

[11] H. Lev-Ari and T. Kailath, "Autoregressive models for nonstationary discrete time processes," in IEEE Int. Symp. Informtion Theory, Cambridge, MA, Aug. 1984.

[12] H. Lev-Ari, S. R. Parker, and T. Kailath, "Multidimensional maximumentropy covariance extension," IEEE Trans. Inform. Theory, vol. 35, pp. 497-508, May 1989.

[13] H. L. Van Trees, Detection, Estimation, and Modulation Theory. New York: Wiley, 1968, vol. I.

[14] L. L. Scharf, Statistical Signal Processing: Detection, Estimation and Time Series Analysis. Reading, MA: Addison-Wesley, 1991.

[15] T. J. Rivlin, The Chebyshev Polynomials. New York: Wiley, 1974. 